\title{
EULERIAN FORMULATION AND LEVEL SET MODELS FOR INCOMPRESSIBLE FLUID-STRUCTURE INTERACTION
}

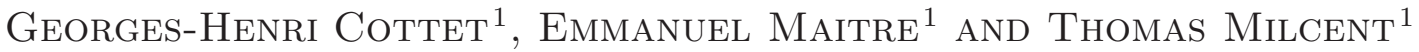

\begin{abstract}
This paper is devoted to Eulerian models for incompressible fluid-structure systems. These models are primarily derived for computational purposes as they allow to simulate in a rather straightforward way complex 3D systems. We first analyze the level set model of immersed membranes proposed in [Cottet and Maitre, Math. Models Methods Appl. Sci. 16 (2006) 415-438]. We in particular show that this model can be interpreted as a generalization of so-called Korteweg fluids. We then extend this model to more generic fluid-structure systems. In this framework, assuming anisotropy, the membrane model appears as a formal limit system when the elastic body width vanishes. We finally provide some numerical experiments which illustrate this claim.
\end{abstract}

Mathematics Subject Classification. 76D05, 74B20, 74F10.

Received July 25, 2007. Revised December 20, 2007.

Published online April 1st, 2008.

\section{INTRODUCTION}

Fluid-structure interaction problems remain among the most challenging problems in computational mechanics. The difficulties in the simulation of these problems stem form the fact that they rely on the coupling of models of different nature: Lagrangian in the solid and Eulerian in the fluid. The so-called ALE (for Arbitrary Lagrangian Eulerian) methods cope with this difficulty by adapting the fluid solver along the deformations of the solid medium in the direction normal to the interface. These methods allow to accurately account for continuity of stresses and velocities at the interface but they are difficult to implement and time-consuming in particular for 3D systems undergoing large deformations. In [4,5], motivated by computational considerations, we introduced and validated an approach based on level set methods to handle elastic membranes interacting with incompressible fluids. The method is in the spirit of the Immersed Boundary Method [15] in the sense that the membrane is accounted for through a force term in the flow equations. However, instead of being tracked in a Lagrangian manner, the membrane is implicitly captured as the zero isosurface of a level set function. The resulting model is proved, both theoretically and numerically, to exhibit satisfactory energy and mass conservation properties.

The purpose of this paper is three-fold. First we provide a mathematical analysis of the membrane-fluid model of $[4,5]$. We prove in Section 2 local existence of strong solutions in 3D. In passing we also observe

\footnotetext{
Keywords and phrases. Fluid structure interaction, elastic membrane, Eulerian method, level set method, Korteweg fluid, Navier-Stokes equations.

${ }^{1}$ Laboratoire Jean Kuntzmann, Université de Grenoble and CNRS, BP 53, 8041 Grenoble Cedex 9, France.

georges-henri.cottet@imap.fr
} 
that, from a mathematical point of view, this model can be seen as a generalization of Korteweg fluid models. Secondly, we extend in Section 3 the fluid-membrane model to handle generic incompressible elastic bodies interacting with $3 \mathrm{D}$ incompressible flows.

The model heavily relies on an Eulerian description of elasticity in the spirit of Ogden [1,13]. The elastic stresses are represented by 3 level set functions and the fluid-structure interface by one additional level set function. We consider first the case of isotropic elasticity and then devote some attention to the case of anisotropic elastic bodies, as they are of interest in many applications in biomechanics that motivated the present work. The use of the gradient of a level set function for the preferred direction leads to a stress formula which is naturally accommodated in a level set formulation and where each term has a clear mechanical meaning, in contrast with usual formulation $[7,13]$ of anisotropic elasticity.

Finally, we investigate, in dimension 2, the case of a thin elastic body, with transverse isotropy, and formally recover the membrane model as the thickness of the body tends to zero. Section 4 is devoted to concluding remarks. The present paper is concerned with mathematical analysis and modeling issues. Application of the models herein described for biological membranes and tissues are given in [6,9].

\section{AnAlysis of A LEVEL-SET MODEL OF IMMERSED ElAStiC MEMBRANES}

In this section we consider an elastic hypersurface immersed in an inhomogeneous viscous incompressible fluid with density $\rho$ and viscosity $\mu$, which flows during a time interval $[0, T], T>0$, in a bounded domain $\Omega$ of $\mathbb{R}^{d}, d=2$ or 3 . Let $u$ be the divergence-free velocity field of the continuous medium. For simplicity, the boundary conditions are either Dirichlet, $u=0$ on $\partial \Omega$, or periodic and in this case $\Omega$ is assumed to be a parallelepiped. We first recall the fluid-structure interaction model derived in $[4,5]$. We reformulate the elastic force in divergence form and we proceed to its mathematical analysis.

\subsection{Level-set formulation}

In order to describe the interface we choose a level set representation following [4]. Let $\varphi_{0}$ be the signed distance to the initial interface $\Gamma_{0}$. Then the solution of the scalar transport equation

$$
\left.\varphi_{t}+u \cdot \nabla \varphi=0 \text { on } \Omega \times\right] 0, T\left[, \quad \varphi=\varphi_{0} \text { on } \Omega \times\{0\}\right.
$$

gives us a representation of the interface at time $t$ as

$$
\Gamma_{t}=\{x \in \Omega, \quad \varphi(x, t)=0\}
$$

While this representation is now classical [14], our contribution was to remark that the solution of (2.1) carries some mechanical information, through $|\nabla \varphi|$. Indeed this quantity, which is solution of

$$
\left.|\nabla \varphi|_{t}+u \cdot \nabla|\nabla \varphi|=-|\nabla \varphi| \frac{\nabla \varphi^{T} \nabla u \nabla \varphi}{|\nabla \varphi|^{2}}=-|\nabla \varphi| \frac{\nabla \varphi \otimes \nabla \varphi}{|\nabla \varphi|^{2}}: \nabla u \text { on } \Omega \times\right] 0, T[
$$

represents the stretching (of the immersed curve in $\mathbb{R}^{2}$ ) or the area change (of the surface in $\mathbb{R}^{3}$ ). In $(2.2$ ) we denoted by $a \otimes b$, for two vectors $a, b$, the tensor $\left(a_{i} b_{j}\right)_{i, j=1 \ldots d}$ and by $A: B$, for two tensors $A, B$ the scalar $A_{i j} B_{i j}$ (summation on repeated indices is assumed).

In the case where the initial immersed surface $\Gamma_{0}$ is stretched by a factor $r$, then $\varphi_{0}$ is taken to be $r$ times the signed distance to $\Gamma_{0}$.

The simplest model for an elastic hypersurface is to consider an energy which only depends on this stretching, and on the interface position. A general energy is given by

$$
\widetilde{\mathcal{E}}_{s}=\int_{\Omega} \alpha(\varphi,|\nabla \varphi|) \mathrm{d} x
$$


The analysis below applies to the above energy. However, since the elastic medium is localized on the interface, it is convenient to introduce a cut-off function $\zeta$ and to consider an energy under the form $[4,5]$

$$
\mathcal{E}_{s}=\int_{\Omega} E(|\nabla \varphi|) \frac{1}{\varepsilon} \zeta\left(\frac{\varphi}{\varepsilon}\right) \mathrm{d} x
$$

An example of a commonly used cut-off function is the function vanishing outside $[-1,1]$ and defined by $\zeta(r)=\frac{1}{2}(1+\cos \pi r)$ for $|r|<1$.

\subsection{Divergence form of the elastic force}

Taking formally the time derivative of $\mathcal{E}_{s}$ leads to

$$
\frac{\mathrm{d} \mathcal{E}_{s}}{\mathrm{~d} t}=\int_{\Omega} E^{\prime}(|\nabla \varphi|)|\nabla \varphi|_{t} \frac{1}{\varepsilon} \zeta\left(\frac{\varphi}{\varepsilon}\right)+E(|\nabla \varphi|) \frac{1}{\varepsilon^{2}} \zeta^{\prime}\left(\frac{\varphi}{\varepsilon}\right) \varphi_{t} \mathrm{~d} x
$$

Using (2.1) and (2.2) gives

$$
\frac{\mathrm{d} \mathcal{E}_{s}}{\mathrm{~d} t}=-\int_{\Omega}\left(E^{\prime}(|\nabla \varphi|)|\nabla \varphi| \frac{\nabla \varphi \otimes \nabla \varphi}{|\nabla \varphi|^{2}}: \nabla u+E^{\prime}(|\nabla \varphi|) u \cdot \nabla|\nabla \varphi|\right) \frac{1}{\varepsilon} \zeta\left(\frac{\varphi}{\varepsilon}\right)+E(|\nabla \varphi|) \frac{1}{\varepsilon^{2}} \zeta^{\prime}\left(\frac{\varphi}{\varepsilon}\right) u \cdot \nabla \varphi \mathrm{d} x .
$$

If we integrate by parts the first term (the boundary term cancels thanks to homogeneous or periodic boundary conditions) and combine the two others we get

$$
\frac{\mathrm{d} \mathcal{E}_{s}}{\mathrm{~d} t}=-\int_{\Omega}-\operatorname{div}\left(E^{\prime}(|\nabla \varphi|)|\nabla \varphi| \frac{\nabla \varphi \otimes \nabla \varphi}{|\nabla \varphi|^{2}} \frac{1}{\varepsilon} \zeta\left(\frac{\varphi}{\varepsilon}\right)\right) \cdot u+\nabla\left(E(|\nabla \varphi|) \frac{1}{\varepsilon} \zeta\left(\frac{\varphi}{\varepsilon}\right)\right) \cdot u \mathrm{~d} x .
$$

This expression stands for the power of the elastic force which may therefore be written as

$$
F_{s}(x, t)=\nabla\left(E(|\nabla \varphi|) \frac{1}{\varepsilon} \zeta\left(\frac{\varphi}{\varepsilon}\right)\right)-\operatorname{div}\left(E^{\prime}(|\nabla \varphi|)|\nabla \varphi| \frac{\nabla \varphi \otimes \nabla \varphi}{|\nabla \varphi|^{2}} \frac{1}{\varepsilon} \zeta\left(\frac{\varphi}{\varepsilon}\right)\right) .
$$

Note that this force is defined up to a gradient term, since $u$ is divergence free. This divergence form of the elastic force is thus equivalent to the following expression derived in [4] from a classical Lagrangian representation of the interface:

$$
F_{s}(x, t)=\left\{\nabla\left[E^{\prime}(|\nabla \varphi|)\right]-\operatorname{div}\left[E^{\prime}(|\nabla \varphi|) \frac{\nabla \varphi}{|\nabla \varphi|}\right] \frac{\nabla \varphi}{|\nabla \varphi|}\right\}|\nabla \varphi| \frac{1}{\varepsilon} \zeta\left(\frac{\varphi}{\varepsilon}\right) .
$$

This force has support on a subset of $\Gamma_{t}^{\varepsilon}=\{x \in \Omega,|\varphi(x, t)|<\varepsilon\}$. At $t=0, \varphi_{0}$ is a scaled signed distance function, thus $\Gamma_{0}^{\varepsilon}$ has a width of $\frac{2 \varepsilon}{r}$ where $r$ is the initial stretching. However $\varphi(t)$, for $t>0$, as solution of (2.1), does not remain a distance function. Thus the width of $\Gamma_{t}^{\varepsilon}$ depends on the local stretching, which is not desirable for accuracy reasons. A simple way to overcome this difficulty is to re-normalize $\varphi$ by its gradient (or, equivalently, to replace $\varepsilon$ by $|\nabla \varphi| \varepsilon$ ), in order to recover to first-order distance function. While this seems a rather crude approximation, several validation studies $[5,6]$ indicate good behaviour in comparison with the usual reinitialization step performed in level-set methods [14], and with other approximations of the Dirac function proposed in [16].

Note that this post-processing is made numerically only in the final expression (2.6) of the force, thus there is no hope that this force could derive from an energy. Nevertheless, one could seek which force a renormalized energy would lead to. More generally, we could have worked on the energy (2.3). In that case, arguing as for $\mathcal{E}_{s}$, one gets

$$
\widetilde{F}_{s}=\nabla(\alpha(\varphi,|\nabla \varphi|))-\operatorname{div}\left(\frac{\partial \alpha}{\partial|\nabla \varphi|}(\varphi,|\nabla \varphi|) \frac{\nabla \varphi \otimes \nabla \varphi}{|\nabla \varphi|}\right) .
$$


We recover $F_{s}$ by setting $\alpha(\varphi,|\nabla \varphi|)=E(|\nabla \varphi|) \frac{1}{\varepsilon} \zeta\left(\frac{\varphi}{\varepsilon}\right)$, while the normalized case corresponds to the choice $\alpha(\varphi,|\nabla \varphi|)=\frac{E(|\nabla \varphi|)}{|\nabla \varphi|} \frac{1}{\varepsilon} \zeta\left(\frac{\varphi}{\varepsilon|\nabla \varphi|}\right)$. Inserting this expression into (2.7) gives

$$
\begin{aligned}
\frac{\partial \alpha}{\partial|\nabla \varphi|}(\varphi,|\nabla \varphi|) & =\frac{E^{\prime}(|\nabla \varphi|)}{|\nabla \varphi|} \frac{1}{\varepsilon} \zeta\left(\frac{\varphi}{\varepsilon|\nabla \varphi|}\right) \\
& -\frac{E(|\nabla \varphi|)}{|\nabla \varphi|^{2}}\left[\frac{1}{\varepsilon} \zeta\left(\frac{\varphi}{\varepsilon|\nabla \varphi|}\right)+\frac{\varphi}{\varepsilon|\nabla \varphi|} \frac{1}{\varepsilon} \zeta^{\prime}\left(\frac{\varphi}{\varepsilon|\nabla \varphi|}\right)\right] \\
& =\frac{E^{\prime}(|\nabla \varphi|)}{|\nabla \varphi|} \frac{1}{\varepsilon} \zeta\left(\frac{\varphi}{\varepsilon|\nabla \varphi|}\right)-\frac{E(|\nabla \varphi|)}{|\nabla \varphi|^{2}} \frac{1}{\varepsilon}(r \zeta(r))^{\prime}\left(\frac{\varphi}{\varepsilon|\nabla \varphi|}\right)
\end{aligned}
$$

thus leading to

$$
\widetilde{F}_{s}=\nabla\left[\frac{E(|\nabla \varphi|)}{|\nabla \varphi|} \frac{1}{\varepsilon} \zeta\left(\frac{\varphi}{\varepsilon|\nabla \varphi|}\right)\right]-\operatorname{div}\left(\frac{E^{\prime}(|\nabla \varphi|)}{|\nabla \varphi|} \frac{\nabla \varphi \otimes \nabla \varphi}{|\nabla \varphi|} \frac{1}{\varepsilon} \zeta\left(\frac{\varphi}{\varepsilon|\nabla \varphi|}\right)-R\right)
$$

which is exactly (2.5) with $\varepsilon|\nabla \varphi|$ in place of $\varepsilon$, up to the residual term

$$
R=\frac{E(|\nabla \varphi|)}{|\nabla \varphi|^{2}} \frac{1}{\varepsilon}(r \zeta(r))^{\prime}\left(\frac{\varphi}{\varepsilon|\nabla \varphi|}\right) \frac{\nabla \varphi \otimes \nabla \varphi}{|\nabla \varphi|}
$$

Note that $r \rightarrow-r \zeta^{\prime}(r)$ is a cutoff function since $\int_{\mathbb{R}}-r \zeta^{\prime}(r) \mathrm{d} r=\int_{\mathbb{R}} \zeta(r) \mathrm{d} r$. Thus $r \rightarrow(r \zeta(r))^{\prime}$ has compact support and its integral on $\mathbb{R}$ vanishes.

Remark 2.1. In some applications where this model is used to obtain equilibrium shapes in biological vesicles [6], curvature forces are to be taken into account. This corresponds to the curvature energy:

$$
\mathcal{E}_{c}=\int_{\Omega} G(\kappa(\varphi))|\nabla \varphi| \frac{1}{\varepsilon} \zeta\left(\frac{\varphi}{\varepsilon}\right) \mathrm{d} x
$$

where $\kappa(\varphi)$ is the curvature, given in terms of $\varphi$ by $\kappa(\varphi)=\operatorname{div} \frac{\nabla \varphi}{|\nabla \varphi|}$. It is shown in [10] that differentiating this energy leads to the following expression of the force:

$$
F_{c}=\operatorname{div}\left[-G(\kappa(\varphi)) \frac{\nabla \varphi}{|\nabla \varphi|}+\frac{1}{|\nabla \varphi|} \mathbb{P}_{\nabla \varphi^{\perp}}\left[\nabla\left(|\nabla \varphi| G^{\prime}(\kappa(\varphi))\right)\right]\right] \frac{1}{\varepsilon} \zeta\left(\frac{\varphi}{\varepsilon}\right) \nabla \varphi
$$

\subsection{Fluid-structure model}

In this paragraph we restrict ourselves to the case of two homogeneous incompressible fluids of densities $\rho_{1}$ and $\rho_{2}$, separated by an elastic interface of surface density $\lambda_{\theta}$ in a reference configuration. Let $G(r)=$ $\int_{-\infty}^{r} \zeta(s) \mathrm{d} s$, and $\rho_{\varepsilon}(\varphi)=\rho_{1}+G\left(\frac{\varphi}{\varepsilon}\right)\left(\rho_{2}-\rho_{1}\right)+\lambda_{\theta} \frac{1}{\varepsilon} \zeta\left(\frac{\varphi}{\varepsilon}\right)$. Similarly if we consider inner and outer fluid viscosities $\mu_{1}$ and $\mu_{2}$, we define a global viscosity through $\mu_{\varepsilon}(\varphi)=\mu_{1}+G\left(\frac{\varphi}{\varepsilon}\right)\left(\mu_{2}-\mu_{1}\right)$.

Our fluid-structure interaction problem then turns into the following flow problem: find $(u, \varphi)$ solution in $\Omega \times] 0, T[$ of

$$
\left\{\begin{array}{l}
\rho_{\varepsilon}(\varphi)\left(u_{t}+u \cdot \nabla u\right)-\operatorname{div}\left(\mu_{\varepsilon}(\varphi) D(u)\right)+\nabla p=F_{s}(x, t) \\
\operatorname{div} u=0 \\
\varphi_{t}+u \cdot \nabla \varphi=0 .
\end{array}\right.
$$


Note that this regularization of the fluid-structure problem does not introduce energy dissipation. Indeed we recall the following energy equality proved in [5]:

$$
\begin{array}{r}
\frac{1}{2} \int_{\Omega} \rho_{\varepsilon}(\varphi) u^{2}(x, t) \mathrm{d} x+\int_{\Omega} E(|\nabla \varphi|) \frac{1}{\varepsilon} \zeta\left(\frac{\varphi}{\varepsilon}\right) \mathrm{d} x+\int_{0}^{t} \int_{\Omega} \mu_{\varepsilon}(\varphi)|\nabla u|^{2} \mathrm{~d} x= \\
\frac{1}{2} \int_{\Omega} \rho_{\varepsilon}(\varphi) u_{0}^{2}(x) \mathrm{d} x+\int_{\Omega} E\left(\left|\nabla \varphi_{0}\right|\right) \frac{1}{\varepsilon} \zeta\left(\frac{\varphi}{\varepsilon}\right) \mathrm{d} x .
\end{array}
$$

This identity is the starting estimate in the mathematical analysis which follows.

\subsection{Mathematical analysis}

For a sake of simplicity we fix $\epsilon=1, \rho_{1}=\rho_{2}, \mu_{1}=\mu_{2}$ and we consider the following model

$$
\begin{aligned}
\rho(\varphi)\left(u_{t}+(u \cdot \nabla) u\right)-\mu \Delta u+\nabla \pi & =-\operatorname{div}(\Sigma(\varphi, \nabla \varphi)) \\
\varphi_{t}+u \cdot \nabla \varphi & =0 \\
\operatorname{div}(u) & =0
\end{aligned}
$$

where

$$
\rho(\varphi)=\bar{\rho}+\lambda \zeta(\varphi)
$$

and $\bar{\rho}, \lambda$ are two strictly positive coefficients (the fluid background and membrane surface densities). $\Sigma$ derives from the energy $E$ of the membrane through the following formula

$$
\Sigma(\varphi, \nabla \varphi)=\frac{E^{\prime}(|\nabla \varphi|)}{|\nabla \varphi|} \zeta(\varphi) \nabla \varphi \otimes \nabla \varphi
$$

which corresponds to (2.5) where the gradient term has been absorbed by the pressure $\pi$. This system is supplemented by initial conditions for $u$ and $\varphi$

$$
u(x, 0)=u_{0}(x), \varphi(x, 0)=\varphi_{0}(x)
$$

and boundary conditions for $u$. We will focus on no-slip boundary conditions (our result easily extends to periodic boundary conditions): $u=0$ on $\partial \Omega$. $\zeta$ denotes a $C^{\infty}$ positive cut-off function. Throughout this section we will assume that

$$
r \rightarrow E^{\prime}(r) \in \mathcal{C}^{1}([0,+\infty)) .
$$

Note that the case of a linear response is given by $E^{\prime}(r)=\lambda(r-1)$ (however the material is still geometrically nonlinear).

Note that our formulation allows to recover Korteweg fluids [18] as a particular case, when $E^{\prime}(r)=r$, the level set function $\varphi$ playing the role of density. Indeed if we choose $E^{\prime}(r)=r$ and if we introduce a function $Z($. such that its derivative is $\sqrt{\zeta(.)}$, setting $\psi=Z(\varphi)$ gives

$$
\zeta(\varphi) \nabla \varphi \otimes \nabla \varphi=\nabla \psi \otimes \nabla \psi .
$$

Clearly, $\psi$ is, like $\varphi$ solution of the transport equation. Moreover, since $\operatorname{div}(\nabla \psi \otimes \nabla \psi)=\Delta \psi \nabla \psi+D^{2} \psi \nabla \psi=$ $\Delta \psi \nabla \psi+\frac{1}{2} \nabla|\nabla \psi|^{2}$, we obtain for the right hand side of the Navier-Stokes equations, up to a gradient term that can be absorbed in the pressure term, the usual Korteweg source term.

In this section we prove the following result:

Theorem 2.2. Let $p>3$. Assume $\Omega$ is a smooth connected bounded domain in $\mathbb{R}^{3}, \varphi_{0} \in W^{2, p}(\Omega)$, such that $\left|\nabla \varphi_{0}\right| \geq \alpha>0$ in a neighborhood of $\left\{\varphi_{0}=0\right\}$, and $u_{0} \in W_{0}^{1, p}(\Omega) \cap W^{2, p}(\Omega)$, with $\operatorname{div} u_{0}=0$. Then there exists 
$T^{*}>0$ only depending on the initial data such that a solution of (2.10), (2.11), (2.12) exists in $\left[0, T^{*}\right]$ with $\varphi \in L^{\infty}\left(0, T^{*} ; W^{2, p}(\Omega)\right), u \in L^{\infty}\left(0, T^{*} ; W_{0}^{1, p}(\Omega)\right) \cap L^{p}\left(0, T^{*} ; W^{2, p}(\Omega)\right)$ and $\nabla \pi \in L^{p}\left(0, T^{*} ; L^{p}(\Omega)\right)$.

Our proof is based on a compactness argument. We classically start by considering a sequence of mollified problems for which smooth solutions are available. We consider a time-retarded mollification of the velocity (see [2], p. 823) and a spatial regularization of the level-set function. Let $\eta$ be a positive parameter and $\xi$ be a positive smooth cut-off function with support in the interval $[1,2]$. For $v \in L^{1}\left(0, T ; W_{0}^{1, p}(\Omega)\right)$ we set

$$
\widetilde{v}^{\eta}=\frac{1}{\eta} \int_{\mathbb{R}} v(\cdot, t-s) \xi\left(\frac{s}{\eta}\right) \mathrm{d} s
$$

where in the above integral $v$ is extended by 0 for negative time. Note that with this definition time-retarded mollification is stable in $L^{p}\left(0, t ; W_{0}^{1, p}(\Omega)\right)$ for any $t>0$. For the spatial regularization of the level-set function we proceed as follows: first, for $\psi \in W^{2, p}(\Omega)$ we denote by $E \psi$ a smooth extension of $\psi$ outside $\Omega$ satisfying $|E \psi|_{W^{2, p}} \leq C|\psi|_{W^{2, p}},|E \psi|_{L^{\infty}} \leq C|\psi|_{L^{\infty}}$ and $|E \psi|_{W^{1, \infty}} \leq C|\psi|_{W^{1, \infty}}$; we then consider a smooth positive, radially symmetric function $\theta$ with support in a ball of radius $1 / 2$. We set $\theta_{\eta}(x)=\eta^{-3} \theta(x / \eta)$. Finally we define

$$
\bar{\psi}^{\eta}=E \psi \star \theta_{\eta} .
$$

With the above notations and definitions we consider the following problem

$$
\begin{aligned}
\rho\left(\bar{\varphi}_{\eta}^{\eta}\right)\left(u_{\eta, t}+\left(\widetilde{u}_{\eta}^{\eta} \cdot \nabla\right) \widetilde{u}_{\eta}^{\eta}\right)-\mu \Delta u_{\eta}+\nabla \pi_{\eta} & =-\operatorname{div}\left(\Sigma\left(\bar{\varphi}_{\eta}^{\eta}, \nabla \bar{\varphi}_{\eta}^{\eta}\right)\right) \\
\varphi_{\eta, t}+\widetilde{u}_{\eta}^{\eta} \cdot \nabla \varphi_{\eta} & =0 \\
\operatorname{div}\left(u_{\eta}\right) & =0 .
\end{aligned}
$$

The existence of global in time solutions $\left(u_{\eta}, \varphi_{\eta}\right)$ for the system (2.19)-(2.21) can easily be proved by induction: for the first time step, by construction of $\widetilde{v}^{\eta}$ our problem amounts to an advection equation with zero velocity combined with a Stokes equation. Next, if, for some integer $k>0$, we assume that

$$
\left(u_{\eta}, \varphi_{\eta}\right) \in L^{p}\left(0, k \eta ; W^{2, p} \cap W_{0}^{1, p}(\Omega)\right) \times L^{\infty}\left(0, k \eta ; W^{2, p}(\Omega)\right),
$$

then $\widetilde{u}_{\eta}^{\eta}$ is by construction in $L^{\infty}\left(k \eta,(k+1) \eta ; W^{2, p}(\Omega)\right)$ and thus from $p>3$ and Sobolev injections,

$$
\widetilde{u}_{\eta}^{\eta} \in L^{\infty}\left(k \eta,(k+1) \eta ; W^{1, \infty}(\Omega)\right) .
$$

Therefore, (2.20) returns a solution in $L^{\infty}\left(k \eta,(k+1) \eta ; W^{2, p}(\Omega)\right)$. As a result, $(2.19)$ is, on the time interval $[k \eta,(k+1) \eta]$, a Stokes equation with right hand side in $L^{\infty}\left(k \eta,(k+1) \eta ; L^{p}(\Omega)\right)$ and has therefore a solution in $L^{p}\left(k \eta,(k+1) \eta ; W^{2, p} \cap W_{0}^{1, p}(\Omega)\right)$. Note that time retarded mollification avoids the technical difficulties related to constructing divergence-free spatial regularization vanishing at the boundary.

To pass to the limit in (2.19), (2.20), (2.21) we clearly need in particular strong convergence on the sequence $\bar{\varphi}_{\eta}^{\eta}$, and thus a priori estimates on second derivatives of $\varphi$. We are going to prove the following result:

Proposition 2.3. There exists a time $T^{*}>0$ and a constant $C$ only depending on $u_{0}$ and $\varphi_{0}$ such that the following estimates hold for $t \leq T^{*}$ :

$$
\left|\varphi_{\eta}(\cdot, t)\right|_{W^{2, p}}+\left|u_{\eta}(\cdot, t)\right|_{W^{1, p}}+\int_{0}^{t}\left|u_{\eta}(\cdot, s)\right|_{W^{2, p}}^{p}+\left|u_{\eta, t}(\cdot, s)\right|_{L^{p}}^{p} \mathrm{~d} s \leq C .
$$


In the sequel for a sake of simplicity we drop the subscripts and superscripts $\eta$. The notation $C$ is used to denote various coefficients only depending on the initial conditions $u_{0}, \varphi_{0}$. Likewise, $G$ will denote various positive, non-decreasing functions on $[0,+\infty)$, only depending on the material law $E^{\prime}(r)$ and domain geometry. When there is no ambiguity we will write $L^{p}, W^{1, p}, W^{2, p}$ instead of $L^{p}(\Omega), W^{1, p}(\Omega), W^{2, p}(\Omega)$.

We consider the conjugate number $q$ of $p(1 / p+1 / q=1)$. Differentiating twice $(2.20)$ we obtain the following transport equation for $\Delta \varphi$ :

$$
\Delta \varphi_{t}+\Delta \widetilde{u} \cdot \nabla \varphi+2[\nabla \widetilde{u}]:\left[D^{2} \varphi\right]+\widetilde{u} \cdot \nabla \Delta \varphi=0 .
$$

Multiplying this equation by $|\Delta \varphi|^{p-2} \Delta \varphi$ and integrating over $\Omega$ yields

$$
\begin{aligned}
& \int_{\Omega} \Delta \varphi_{t}|\Delta \varphi|^{p-2} \Delta \varphi \mathrm{d} x+\int_{\Omega} \Delta \widetilde{u} \cdot \nabla \varphi|\Delta \varphi|^{p-2} \Delta \varphi \mathrm{d} x \\
& \quad+2 \int_{\Omega}[\nabla \widetilde{u}]:\left[D^{2} \varphi\right]|\Delta \varphi|^{p-2} \Delta \varphi \mathrm{d} x+\int_{\Omega} \widetilde{u} \cdot \nabla \Delta \varphi|\Delta \varphi|^{p-2} \Delta \varphi \mathrm{d} x=0 .
\end{aligned}
$$

If we denote by $I_{i}, i=1, \ldots, 4$, the successive terms in the left hand side above, we first have

$$
I_{1}=\frac{1}{p} \frac{\mathrm{d}}{\mathrm{d} t}\left(|\Delta \varphi|_{L^{p}}^{p}\right)
$$

Since $\widetilde{u}=0$ on $\partial \Omega$, we have

$$
I_{4}=\frac{1}{p} \int_{\Omega} \widetilde{u} \cdot \nabla\left(|\Delta \varphi|^{p}\right) \mathrm{d} x=\frac{1}{p} \int_{\partial \Omega}|\Delta \varphi|^{p} \widetilde{u} \cdot n \mathrm{~d} x=0 .
$$

We next easily derive the following estimates

$$
\begin{gathered}
I_{2} \leq\left.\left.|\nabla \varphi|_{L^{\infty}}|\Delta \widetilde{u}|_{L^{p}}|| \Delta \varphi\right|^{p-2} \Delta \varphi\right|_{L^{q}}=|\nabla \varphi|_{L^{\infty}}|\Delta \widetilde{u}|_{L^{p}}|\Delta \varphi|_{L^{p}}^{p-1}, \\
I_{3} \leq\left.\left.|\nabla \widetilde{u}|_{L^{\infty}}\left|D^{2} \varphi\right|_{L^{p}}|| \Delta \varphi\right|^{p-2} \Delta \varphi\right|_{L^{q}} .
\end{gathered}
$$

By Sobolev imbeddings, we have $W^{2, p}(\Omega) \subset W^{1, \infty}(\Omega)$. Furthermore $\varphi \rightarrow\|\varphi\|_{L^{p}}+\|\Delta \varphi\|_{L^{p}}$ is a norm in $W^{2, p}(\Omega)$ (see for instance [19]) and the transport equation (2.20) preserves $L^{p}$ norms. As a result:

$$
|\nabla \varphi|_{L^{\infty}} \leq C\left(1+|\Delta \varphi|_{L^{p}}\right) .
$$

Similarly, $u \rightarrow\|\Delta u\|_{L^{p}}$ is a norm in $W_{0}^{1, p}(\Omega) \cap W^{2, p}(\Omega)$ and therefore, by Sobolev imbedding,

$$
|\nabla \widetilde{u}|_{L^{\infty}} \leq C|\Delta \widetilde{u}|_{L^{p}}
$$

We conclude that

$$
I_{2} \leq C|\Delta \widetilde{u}|_{L^{p}}\left(1+|\Delta \varphi|_{L^{p}}^{p}\right)
$$

and

$$
I_{3} \leq C|\Delta \widetilde{u}|_{L^{p}}|\Delta \varphi|_{L^{p}}^{p}
$$

Combining (2.23), (2.24), (2.25) and (2.26) yields the following estimate

$$
\frac{\mathrm{d}}{\mathrm{d} t}\left(|\Delta \varphi|_{L^{p}}^{p}\right) \leq C|\Delta \widetilde{u}|_{L^{p}}|\Delta \varphi|_{L^{p}}^{p}
$$


Using again the conservation of the $L^{p}$ norm of $\varphi$ we get

$$
\frac{\mathrm{d}}{\mathrm{d} t}\left(|\varphi|_{W^{2, p}}^{p}\right) \leq C|\Delta \widetilde{u}|_{L^{p}}|\Delta \varphi|_{L^{p}}^{p} \leq C\left(|\Delta \widetilde{u}|_{L^{p}}^{p}+|\Delta \varphi|_{L^{p}}^{\frac{p^{2}}{p-1}}\right) .
$$

We now need to derive estimates on the velocity. Viewing (2.19)-(2.21) as a Stokes problem with variable density, we can use the $L^{p}$ estimates established in [17], namely

$$
\begin{aligned}
\int_{0}^{t}\left|u_{t}\right|_{L^{p}}^{p} \mathrm{~d} s+\mu \int_{0}^{t}|\Delta u|_{L^{p}}^{p} \mathrm{~d} s+\int_{0}^{t}|\nabla \pi|_{L^{p}}^{p} \mathrm{~d} s & \\
& P\left(M_{\infty}(t)\right)\left(1+\int_{0}^{t}|(\widetilde{u} \cdot \nabla) \widetilde{u}|_{L^{p}}^{p} \mathrm{~d} s+\int_{0}^{t}|\operatorname{div}(\Sigma(\bar{\varphi},|\nabla \bar{\varphi}|))|_{L^{p}}^{p} \mathrm{~d} s\right)
\end{aligned}
$$

where

$$
M_{\infty}(t)=\|\nabla \rho\|_{L^{\infty}([0, t] \times \Omega)}+\left\|\rho_{t}\right\|_{L^{\infty}([0, t] \times \Omega)}
$$

and $P$ is a non-decreasing polynomial function. By $(2.13)$ we have

$$
M_{\infty}(t) \leq C\left(\|\nabla \bar{\varphi}\|_{L^{\infty}([0, t] \times \Omega)}+\left\|\bar{\varphi}_{t}\right\|_{L^{\infty}([0, t] \times \Omega)}\right)
$$

which, in view of (2.18) and (2.20), gives

$$
M_{\infty}(t) \leq C\left(1+\|\widetilde{u}\|_{L^{\infty}([0, t] \times \Omega)}\right)\|\nabla \varphi\|_{L^{\infty}([0, t] \times \Omega)} .
$$

We now turn to estimating the integral in the right hand side of (2.28). We first write

$$
|(\widetilde{u} \cdot \nabla) \widetilde{u}|_{L^{p}}^{p} \leq C|\nabla \widetilde{u}|_{L^{p}}^{p}|\widetilde{u}|_{L^{\infty}}^{p} \leq C|\nabla \widetilde{u}|_{L^{p}}^{2 p} .
$$

We next use (2.14) and expand the term $\operatorname{div} \Sigma$ to find $\operatorname{div} \Sigma=A_{1}+A_{2}+A_{3}$ where

$$
\begin{gathered}
A_{1}=\zeta(\bar{\varphi})(\nabla \bar{\varphi} \otimes \nabla \bar{\varphi}) \nabla\left(\frac{E^{\prime}(|\nabla \bar{\varphi}|)}{|\nabla \bar{\varphi}|}\right)=\zeta(\bar{\varphi})\left(\frac{\nabla \bar{\varphi}}{|\nabla \bar{\varphi}|} \otimes \frac{\nabla \bar{\varphi}}{|\nabla \bar{\varphi}|}\right)\left(E^{\prime \prime}(|\nabla \bar{\varphi}|) D^{2} \bar{\varphi} \nabla \bar{\varphi}-E^{\prime}(|\nabla \bar{\varphi}|) D^{2} \bar{\varphi} \frac{\nabla \bar{\varphi}}{|\nabla \bar{\varphi}|}\right) \\
A_{2}=\frac{E^{\prime}(|\nabla \bar{\varphi}|)}{|\nabla \bar{\varphi}|} \zeta^{\prime}(\bar{\varphi})(\nabla \bar{\varphi} \otimes \nabla \bar{\varphi}) \nabla \bar{\varphi}=E^{\prime}(|\nabla \bar{\varphi}|)|\nabla \bar{\varphi}| \zeta^{\prime}(\bar{\varphi}) \nabla \bar{\varphi},
\end{gathered}
$$

and

$$
A_{3}=\frac{E^{\prime}(|\nabla \bar{\varphi}|)}{|\nabla \bar{\varphi}|} \zeta(\bar{\varphi}) \operatorname{div}(\nabla \bar{\varphi} \otimes \nabla \bar{\varphi})=E^{\prime}(|\nabla \bar{\varphi}|) \zeta(\bar{\varphi})\left(D^{2} \bar{\varphi} \frac{\nabla \bar{\varphi}}{|\nabla \bar{\varphi}|}+\Delta \bar{\varphi} \frac{\nabla \bar{\varphi}}{|\nabla \bar{\varphi}|}\right)
$$

Straightforward calculations give

$$
\left|A_{1}\right| \leq\left|D^{2} \bar{\varphi}\right|\left(\left|E^{\prime \prime}(|\nabla \bar{\varphi}|)\right||\nabla \bar{\varphi}|+\left|E^{\prime}(|\nabla \bar{\varphi}|)\right|\right) \zeta(\bar{\varphi}) .
$$

For every function $f$ continuous on $[0,+\infty[$ there exists a continuous nondecreasing function $G$ such that $\forall r \in\left[0,+\infty\left[,|f(r)| \leq G(r)\right.\right.$ (e.g. $\left.G(r)=\max _{s \in[0, r]}(|f(s)|)\right)$. Of course $G$ depends on $f$, but from now on we will denote by $G$ a generic nondecreasing function and use it to bound various continuous functions of Sobolev norms of $\bar{\varphi}$ (thus $G$ will depend on $\Omega$ ).

As $r \rightarrow E^{\prime}(r)+r E^{\prime \prime}(r)$ is continuous by assumption (2.16), by Sobolev imbeddings,

$$
\left|A_{1}\right|_{L^{p}} \leq C|\bar{\varphi}|_{W^{2, p}} G\left(|\nabla \bar{\varphi}|_{L^{\infty}}\right) \leq C|\bar{\varphi}|_{W^{2, p}} G\left(C|\bar{\varphi}|_{W^{2, p}}\right) \leq G\left(|\bar{\varphi}|_{W^{2, p}}\right) .
$$

Similarly

$$
A_{2} \leq C|\nabla \bar{\varphi}|^{2}\left|\zeta^{\prime}(\bar{\varphi})\right|\left|E^{\prime}(|\nabla \bar{\varphi}|)\right|
$$


thus

Finally

$$
\left|A_{2}\right|_{L^{p}} \leq G\left(|\nabla \bar{\varphi}|_{L^{\infty}}\right) \leq G\left(|\bar{\varphi}|_{W^{2, p}}\right)
$$

and therefore, as above,

$$
A_{3} \leq C\left|D^{2} \bar{\varphi}\right|\left|E^{\prime}(|\nabla \bar{\varphi}|)\right| \zeta(\bar{\varphi})
$$

Combining (2.31), (2.32), (2.33) and (2.18) yields

$$
\left|A_{3}\right|_{L^{p}}^{p} \leq G\left(|\bar{\varphi}|_{W^{2, p}}\right)
$$

$$
|\operatorname{div} \Sigma|_{L^{p}}^{p} \leq G\left(|\bar{\varphi}|_{W^{2, p}}^{p}\right) \leq G\left(|\varphi|_{W^{2, p}}^{p}\right)
$$

where we recall that $G$ denotes a non-decreasing function. From (2.28), we get the following estimate, for $t>0$

$$
\int_{0}^{t}\left|u_{t}\right|_{L^{p}}^{p} \mathrm{~d} s+\mu \int_{0}^{t}|\Delta u|_{L^{p}}^{p} \mathrm{~d} s+\int_{0}^{t}|\nabla \pi|_{L^{p}}^{p} \mathrm{~d} s \leq P\left(M_{\infty}(t)\right)\left(1+\int_{0}^{t}|\nabla \widetilde{u}|_{L^{p}}^{2 p} \mathrm{~d} s+\int_{0}^{t} G\left(|\Delta \varphi|_{L^{p}}^{p}\right) \mathrm{d} s\right) .
$$

It remains now to estimate $M_{\infty}$ and $|\nabla u|_{L^{p}}$. We start by writing

$$
\frac{1}{p} \frac{\mathrm{d}}{\mathrm{d} t}\left(|\nabla u|_{L^{p}}^{p}\right)=-\int_{\Omega} u_{t} \cdot \nabla\left(\nabla u|\nabla u|^{p-2}\right) \mathrm{d} x
$$

Expanding the integrand in the right hand side one gets

$$
\frac{\mathrm{d}}{\mathrm{d} t}\left(|\nabla u|_{L^{p}}^{p}\right) \leq p|u|_{W^{2, p}}\left|u_{t}\right|_{L^{p}}|\nabla u|_{L^{r}}^{p-2}
$$

where $r$ satisfies $2 / p+1 / r=1$, that is $r=p /(p-2)$, from where we deduce the following estimate

$$
\frac{\mathrm{d}}{\mathrm{d} t}\left(|\nabla u|_{L^{p}}^{p}\right) \leq p|u|_{W^{2, p}}\left|u_{t}\right|_{L^{p}}|\nabla u|_{L^{p}}^{p-2} \leq|u|_{W^{2, p}}^{p-1}\left|u_{t}\right|_{L^{p}}
$$

and finally

$$
\frac{\mathrm{d}}{\mathrm{d} t}\left(|\nabla u|_{L^{p}}^{p}\right) \leq C\left(|u|_{W^{2, p}}^{p}+\left|u_{t}\right|_{L^{p}}^{p}\right)
$$

As for $M_{\infty}$, we use (2.29) and Sobolev imbeddings to obtain

$$
\left.M_{\infty}(t) \leq C\left(1+|\nabla \widetilde{u}|_{L^{\infty}\left(0, t ; L^{p}(\Omega)\right)}\right)|\varphi|_{L^{\infty}\left(0, t ; W^{2, p}(\Omega)\right)}\right) .
$$

We are now in a position to conclude. Integrating (2.27) and (2.36) we get

$$
|\nabla u(t)|_{L^{p}}^{p}+|\varphi(t)|_{W^{2, p}}^{p} \leq C\left(1+\int_{0}^{t}\left[|u|_{W^{2, p}}^{p}+|\widetilde{u}|_{W^{2, p}}^{p}+\left|u_{t}\right|_{L^{p}}^{p}+|\Delta \varphi|_{L^{p}}^{\frac{p^{2}}{p-1}}\right] \mathrm{d} s\right) .
$$

By construction of $\widetilde{u}$ in (2.17) we have

$$
\int_{0}^{t}|\widetilde{u}|_{W^{2, p}}^{p} \mathrm{~d} s \leq \int_{0}^{t}|u|_{W^{2, p}}^{p} \mathrm{~d} s
$$

Combining the above bound with (2.35) we thus may write

$$
|\nabla u(t)|_{L^{p}}^{p}+|\varphi(t)|_{W^{2, p}}^{p} \leq C\left(1+P\left(M_{\infty}(t)\right) \int_{0}^{t} G\left(|\nabla \widetilde{u}(s)|_{L^{p}}^{p}+|\varphi(s)|_{W^{2, p}}^{p}\right) \mathrm{d} s\right.
$$


Let us set

$$
y(t)=\sup _{s \leq t}\left(|\varphi(\cdot, s)|_{W^{2, p}}^{p}+|\nabla u(\cdot, s)|_{L^{p}}^{p}\right) .
$$

By construction of $u_{\eta}$ and $\varphi_{\eta}$ it is clear that $y$ is a continuous function of time. We observe that by construction of $\widetilde{u}$ in (2.17) we can write

and, for non-decreasing function $G$,

$$
|\nabla \widetilde{u}|_{L^{\infty}\left(0, t ; L^{p}(\Omega)\right)} \leq|\nabla u|_{L^{\infty}\left(0, t ; L^{p}(\Omega)\right)}
$$

$$
\int_{0}^{t} G\left(|\nabla \widetilde{u}(s)|_{L^{p}}^{p}\right) \mathrm{d} s \leq t G\left(\sup _{s \leq t}|\nabla u(s)|_{L^{p}}^{p}\right) \mathrm{d} s .
$$

In view of (2.37) and (2.38) we therefore get (restoring the $\eta$ dependence)

$$
y_{\eta}(t) \leq C\left(1+G\left(y_{\eta}(t)\right) t\right)
$$

where, again, $G$ is a continuous non-decreasing function depending on the initial condition $u_{0}, \varphi_{0}$ and the domain $\Omega$. As a result, from the continuity with respect to time of $y_{\eta}(t)$, there exists a strictly positive finite time $T^{*}$ and a constant $C$ only depending on the initial condition (and not on $\eta$ ) such that $y_{\eta}(t) \leq C$ for $t \leq T^{*}$. The desired estimate (2.22) follows by combining (2.39) and (2.28).

To summarize, we have established the following a priori estimates:

$$
\begin{aligned}
\text { the sequence } u_{\eta} & \text { is bounded in } L^{\infty}\left(0, T^{*} ; W_{0}^{1, p}\right) \cap L^{p}\left(0, T^{*} ; W^{2, p}\right) \\
\text { the sequence } u_{\eta, t} & \text { is bounded in } L^{p}\left(0, T^{*} ; L^{p}\right) \\
\text { the sequence } \varphi_{\eta} & \text { is bounded in } L^{\infty}\left(0, T^{*} ; W^{2, p}\right) .
\end{aligned}
$$

Using equation (2.20) and

$$
|(u \cdot \nabla) \varphi|_{L^{p}} \leq|u|_{L^{\infty}}|\nabla \varphi|_{L^{p}} \leq|u|_{W^{1, p}}|\nabla \varphi|_{L^{p}}
$$

we also have that $\varphi_{\eta, t}$ is bounded in $L^{\infty}\left(0, T^{*} ; L^{p}\right)$. By classical compactness results we thus conclude that there exists $\varphi \in L^{\infty}\left(0, T^{*} ; W^{2, p}\right)$ and $u \in L^{\infty}\left(0, T^{*} ; W_{0}^{1, p}\right) \cap L^{p}\left(0, T^{*} ; W^{2, p}\right)$ such that

$$
u_{\eta} \rightarrow u \text { in } L^{p}\left(0, T ; W^{1, p}\right) ; \varphi_{\eta} \rightarrow \varphi \text { in } L^{p}\left(0, T ; W^{1, p}\right)
$$

as $\eta \rightarrow 0$. We next write

$$
\widetilde{u}_{\eta}^{\eta}-u=\widetilde{u}_{\eta}^{\eta}-u_{\eta}+u_{\eta}-u .
$$

By construction of $\widetilde{u}_{\eta}^{\eta}$ we get, for $2 \eta \leq t \leq T^{*}$ :

$$
\widetilde{u}_{\eta}^{\eta}(\cdot, t)-u_{\eta}(\cdot, t)=\eta^{-1} \int_{0}^{t}\left(u_{\eta}(\cdot, t-s)-u_{\eta}(\cdot, t)\right) \xi\left(\frac{s}{\eta}\right) \mathrm{d} s
$$

and thus

Since we also clearly have

$$
\left|\widetilde{u}_{\eta}^{\eta}-u_{\eta}\right|_{L^{p}\left(2 \eta, T^{*} ; L^{p}\right)} \leq C \eta\left|u_{\eta, t}\right|_{L^{p}\left(0, T^{*} ; L^{p}\right)} .
$$

we deduce from (2.43) that

$$
\left|\widetilde{u}_{\eta}^{\eta}-u_{\eta}\right|_{L^{1}\left(0,2 \eta ; L^{p}\right)} \leq C \eta^{1-1 / p}\left|u_{\eta}\right|_{L^{p}\left(0, T^{*} ; L^{p}\right)}
$$

$$
\widetilde{u}_{\eta}^{\eta} \rightarrow u \text { in } L^{1}\left(\left[0, T^{*}\right] \times \Omega\right) .
$$

To pass to the limit in the nonlinear terms of (2.19), we rewrite $\left(\widetilde{u}_{\eta}^{\eta} \cdot \nabla\right) \widetilde{u}_{\eta}^{\eta}$ as $\operatorname{div}\left(\widetilde{u}_{\eta}^{\eta} \otimes \widetilde{u}_{\eta}^{\eta}\right)$. Since, by (2.40), $\widetilde{u}_{\eta}^{\eta}$ is bounded in $L^{\infty}\left(\left[0, T^{*}\right] \times \Omega\right)$ we obtain

$$
\widetilde{u}_{\eta}^{\eta} \otimes \widetilde{u}_{\eta}^{\eta} \rightarrow u \otimes u \text { in the sense of distributions. }
$$


Similar arguments show that

$$
\bar{\varphi}_{\eta}^{\eta} \rightarrow \varphi \text { in } L^{p}\left(0, T^{*} ; W^{1, p}(\Omega)\right) \text { and a.e. }
$$

which, by the smoothness of $\Sigma$ and the fact that, due to $(2.42), \nabla \bar{\varphi}_{\eta}^{\eta}$ is bounded in $L^{\infty}\left(\left[0, T^{*}\right] \times \Omega\right)$, in turn implies that

Similarly

$$
\Sigma\left(\bar{\varphi}_{\eta}^{\eta}, \nabla \bar{\varphi}_{\eta}^{\eta}\right) \rightarrow \Sigma(\varphi, \nabla \varphi) \text { in } L^{p}\left(0, T, L^{p}(\Omega)\right)
$$

$$
\rho\left(\bar{\varphi}_{\eta}^{\eta}\right) \rightarrow \rho(\varphi) \text { in } L^{p}\left(0, T, L^{p}(\Omega)\right)
$$

Since, by $(2.13), \rho\left(\bar{\varphi}^{\eta}\right) \geq \bar{\rho}>0$ we deduce that

$$
\frac{\Sigma\left(\bar{\varphi}_{\eta}^{\eta}, \nabla \bar{\varphi}^{\eta}\right)}{\rho\left(\bar{\varphi}_{\eta}^{\eta}\right)} \rightarrow \frac{\Sigma(\varphi, \nabla \varphi)}{\rho(\varphi)} \text { in } L^{p}\left(0, T, L^{p}(\Omega)\right)
$$

Combining the above limit with (2.44) shows that $(u, \varphi)$ is solution to (2.10). Passing to the limits in the other non-linear terms of (2.11) follows similar lines. This concludes the proof that $(u, \varphi)$ is a solution to the system (2.10)-(2.12).

\section{INTERACTION BETWEEN INCOMPRESSIBLE FLUID AND ELASTIC BODY: THE GENERAL CASE}

In this section our goal is to extend the Eulerian model just analyzed to the case of a generic elastic incompressible body immersed in an incompressible 3D fluid. For a sake of simplicity, we first consider a structure occupying the whole computational domain. We will consider the interaction with a fluid in Section 3.3.

Let us first review some classical notions regarding the Eulerian formulation of elasticity. As the incompressible velocity field vanishes on $\partial \Omega$, we are in the special case where the reference domain coincides with the deformed one. Let $\tau \rightarrow X(\tau ; x, t)$ be the forward characteristics of $u$. For the sake of readability, we set $X(\xi, t):=X(t ; \xi, 0)$. We also consider $Y(x, t):=X(0 ; x, t)$, the backward trajectories. One key point in our approach is to observe that these trajectories satisfy the following vector transport equation

$$
Y_{t}+u \cdot \nabla Y=0, \quad Y(0)=x
$$

An hyperelastic material has an energy function depending only on $\nabla X$,

$$
E=\int_{\Omega} \bar{W}(\nabla X)(\xi, t) \mathrm{d} \xi
$$

As $\nabla Y(x, t) \nabla X(\xi, t)=\mathbb{I}$ whenever $\xi=Y(x, t)$, and $X(\Omega, t)=\Omega$, we have

$$
E=\int_{\Omega} \bar{W}\left(\nabla Y^{-1}\right)(x, t) J(x, t) \mathrm{d} x
$$

where $J(x, t)=\operatorname{det} \nabla Y(x, t)$. Note that, for an incompressible elastic material, $J=1$, and we could thus drop the $J$ from the energy expression. However, the expressions obtained by differentiating with respect to time the stored energy will vary (by a gradient term) depending on the presence of $J$.

This energy verifies the frame indifference axiom [12] whenever $\bar{W}(Q F)=\bar{W}(F)$ for all rotations $Q$. Using the polar factorization of $F$ leads to $\bar{W}(F)=\bar{W}\left(C^{\frac{1}{2}}\right)=\widehat{W}(C)$ where $C=F^{T} F$. The Lagrangian tensor $C$ is expressed in terms of $X$ or $Y$ as

$$
C(\xi)=\nabla X^{T} \nabla X(\xi)=\nabla Y^{-T} \nabla Y^{-1}(x), \text { with } x=X(t ; \xi, 0) .
$$

In the sequel we first consider the case of an isotropic medium, and then turn to the case of transverse isotropy. 


\subsection{The isotropic case}

In the isotropic case $\widehat{W}(C)$ depends only on the invariants of $C$ [3], which are the same as those of $B=$ $\nabla Y^{-1} \nabla Y^{-T}$. This last tensor is Eulerian, in the sense that it is solution to the following transport equations

$$
B_{t}+u \cdot \nabla B=\nabla u B+B \nabla u^{T} \quad B_{t}^{-1}+u \cdot \nabla B^{-1}=-\nabla u^{T} B^{-1}-B^{-1} \nabla u .
$$

These equations are easily deduced from the following differential identities:

$$
\begin{gathered}
\left(\nabla Y^{-1}\right)_{t}+u \cdot \nabla\left(\nabla Y^{-1}\right)=\nabla u \nabla Y^{-1} \quad(\nabla Y)_{t}+u \cdot \nabla(\nabla Y)=-\nabla Y \nabla u \\
\left(\nabla Y^{-T}\right)_{t}+u \cdot \nabla\left(\nabla Y^{-T}\right)=\nabla Y^{-T} \nabla u^{T} \quad\left(\nabla Y^{T}\right)_{t}+u \cdot \nabla\left(\nabla Y^{T}\right)=-\nabla u^{T} \nabla Y^{T} .
\end{gathered}
$$

Due to the incompressibility assumption, the invariants of $B$ are, in $\mathbb{R}^{3}, \operatorname{tr} B=\left|\nabla Y^{-1}\right|^{2}$ and $\operatorname{tr} B^{-1}=|\nabla Y|^{2}$, thus the stored energy may be written as

$$
E=\frac{1}{2} \int_{\Omega} W\left(\operatorname{tr} B, \operatorname{tr} B^{-1}\right) \mathrm{d} x
$$

where $(a, b) \rightarrow W(a, b)$ is a given function. Note that, from (3.3), we have

$$
\begin{aligned}
(\operatorname{tr} B)_{t}+u \cdot \nabla(\operatorname{tr} B) & =2 \operatorname{tr}(\nabla u B)=2 B: \nabla u, \\
\left(\operatorname{tr} B^{-1}\right)_{t}+u \cdot \nabla\left(\operatorname{tr} B^{-1}\right) & =-2 \operatorname{tr}\left(B^{-1} \nabla u\right)=-2 B^{-1}: \nabla u .
\end{aligned}
$$

Taking the time derivative of $E$ and using (3.6) gives

$$
\begin{aligned}
\frac{\mathrm{d} E}{\mathrm{~d} t} & =\frac{1}{2} \int_{\Omega} \frac{\partial W}{\partial a}(\operatorname{tr} B)_{t}+\frac{\partial W}{\partial b}\left(\operatorname{tr}\left(B^{-1}\right)\right)_{t} \mathrm{~d} x \\
& =\frac{1}{2} \int_{\Omega} \frac{\partial W}{\partial a}(-u \cdot \nabla(\operatorname{tr} B))+\frac{\partial W}{\partial b}\left(-u \cdot \nabla\left(\operatorname{tr} B^{-1}\right)\right)+\frac{\partial W}{\partial a} 2 B: \nabla u-\frac{\partial W}{\partial b} 2 B^{-1}: \nabla u \mathrm{~d} x \\
& =\int_{\Omega}-\frac{1}{2} u \cdot \nabla W+\left(\frac{\partial W}{\partial a} B-\frac{\partial W}{\partial b} B^{-1}\right): \nabla u \mathrm{~d} x \\
& =\int_{\Omega}\left[-\frac{1}{2} \nabla W-\operatorname{div}\left(\frac{\partial W}{\partial a} B-\frac{\partial W}{\partial b} B^{-1}\right)\right] \cdot u \mathrm{~d} x .
\end{aligned}
$$

This expression should equal minus the power of stress forces, that is $-\int_{\Omega} \operatorname{div} \sigma \cdot u \mathrm{~d} x$. Therefore the incompressibility assumption leads to the following stress:

$$
\sigma=-p \mathbb{I}+\frac{\partial W}{\partial a}\left(\operatorname{tr} B, \operatorname{tr} B^{-1}\right) B-\frac{\partial W}{\partial b}\left(\operatorname{tr} B, \operatorname{tr} B^{-1}\right) B^{-1} .
$$

Note that thanks to Cayley-Hamilton theorem, $B^{-1}=\operatorname{tr} B^{-1} \mathbb{I}-(\operatorname{tr} B) B+B^{2}$. Therefore, up to a gradient term which is absorbed in the pressure, $B^{-1}$ could be replaced by $(\operatorname{tr} B) B-B^{2}$, as in [13]. The Eulerian formulation of isotropic elasticity we just presented is summarized in the following proposition:

Proposition 3.1. The deformation of an isotropic elastic material in Eulerian coordinates is governed by the following equations:

$$
\begin{cases}\rho\left(u_{t}+u \cdot \nabla u\right)-\operatorname{div}\left(\alpha_{1} B-\alpha_{2} B^{-1}\right)+\nabla p=f, & \text { on } \Omega \times] 0, T[, \\ \operatorname{div} u=0, & \text { on } \Omega \times] 0, T[, \\ Y_{t}+u \cdot \nabla Y=0, \quad B=\nabla Y^{-1} \nabla Y^{-T}, & \text { on } \Omega \times] 0, T[, \\ u=0, & \text { on } \partial \Omega \times] 0, T[, \\ u=u_{0}, \quad Y=i d, & \text { on } \Omega \times\{0\} .\end{cases}
$$


where the $\alpha_{i}$ are functions of $\left(\operatorname{tr} B, \operatorname{tr} B^{-1}\right)$ defined as partial derivatives of an energy function $W$.

The following remark is important from the computational point of view. We recall that

$$
B=\nabla Y^{-1} \nabla Y^{-T}=\nabla X \nabla X^{T}=\sum_{i=1}^{3} X_{, \xi_{i}} \otimes X_{, \xi_{i}} .
$$

By definition, $\nabla Y_{j} \cdot X_{, \xi_{i}}=\delta_{i j}$. By the incompressibility of the elastic body we have $\operatorname{det} \nabla Y=1$, and thus we have the following simple expression of $X_{, \xi_{i}}$ in the Eulerian domain

$$
X_{, \xi_{1}}=\nabla Y_{2} \times \nabla Y_{3}, \quad X_{, \xi_{2}}=\nabla Y_{3} \times \nabla Y_{1}, \quad X_{, \xi_{3}}=\nabla Y_{1} \times \nabla Y_{2} .
$$

This has two consequences: first it means that the computation of $B$ in the Eulerian model reduces to simple algebraic operations involving the derivatives of $Y$, which keeps the computational complexity of the model at a reasonable level. Secondly, it allows to interpret $B$ as a sum of projections on lines where two different level set functions $Y_{i}$ are constant.

As an example we write the expression of $\alpha_{1}$ and $\alpha_{2}$ in the case of the Saint-Venant Kirchhoff constitutive law $\left(\right.$ in $\mathbb{R}^{3}$ ). The energy density in this case is given by

$$
\frac{\lambda_{1}}{2}(\operatorname{tr} E)^{2}+\lambda_{2} \operatorname{tr} E^{2}, \quad E=\frac{1}{2}(C-\mathbb{I}),
$$

where $\lambda_{1}$ and $\lambda_{2}$ are the Lamé coefficients. It is easy to see that $\operatorname{tr} E=\frac{1}{2} \operatorname{tr}(B-\mathbb{I})$ and $\operatorname{tr} E^{2}=\frac{1}{4} \operatorname{tr}(B-\mathbb{I})^{2}$. Expanding these terms and using the identity $\operatorname{tr}\left(B^{2}\right)=(\operatorname{tr} B)^{2}-2 \operatorname{tr} B^{-1}$ (since $\operatorname{det} B=1$ ) leads to:

$$
W_{S K}(a, b)=\frac{1}{8}\left(\lambda_{1}+2 \lambda_{2}\right) a^{2}-\left(\frac{3 \lambda_{1}}{4}+\frac{\lambda_{2}}{2}\right) a-\frac{\lambda_{2}}{2} b+\frac{9 \lambda_{1}+6 \lambda_{2}}{8} .
$$

This corresponds to the following coefficients in Proposition 3.1:

$$
\alpha_{1}=\frac{\lambda_{1}+2 \lambda_{2}}{4}(\operatorname{tr} B-3)+2 \lambda_{2}, \quad \alpha_{2}=-\frac{\lambda_{2}}{2} .
$$

\subsection{Anisotropic case}

We now turn to the anisotropic case, that is when the elastic body exhibits preferred stretching directions. We have two particular reasons for considering this important case. First this is a case often encountered in biological tissues, an application which was one of the motivations for the present work. As a matter of fact, continuous elastic models in such tissues can generally be seen as an idealization of viscous fluids filled with one or two dimensional fibers. Secondly, thin anisotropic elastic bodies seem to be an appropriate setting if one wishes to recover membrane models in the limit of width tending to zero, a question we will investigate at the end of this section.

\subsubsection{Transversely isotropic elasticity: simple setting}

In order to study the limit model of an incompressible elastic material when its thickness goes to zero, it seems natural to consider that this elastic body has the same symmetries as the zero thickness limit model. We thus consider a transversely isotropic material: let $\tau(y)$ be a preferred direction (at time $t=0$ ) and assume that the material response is indifferent to arbitrary rotations about the direction $\tau$ and by replacement of $\tau$ by $-\tau$. Following [13], this leads to an energy function, which depends not only (in material coordinates) on $\operatorname{tr} C, \operatorname{tr} C^{-1}$, but also on $\tau^{T} C \tau$ and $\tau^{T} C^{2} \tau$. Note first that, due to the Cayley-Hamilton theorem, we can replace this last invariant by $\tau^{T} C^{-1} \tau$. As we will see below, this form has a more direct mechanical meaning. 
It is also more tractable in our level set approach. The energy may thus be written in Lagrangian coordinates under the following form

$$
E=\int_{\Omega} W\left(\operatorname{tr} C, \operatorname{tr} C^{-1}, \tau(y)^{T} C \tau(y), \tau^{T}(y) C^{-1} \tau(y)\right) \mathrm{d} y .
$$

The invariants of $B$ and $C$ are the same. We use the change of variables $y=Y(x, t)$ with unit Jacobian (due to incompressibility), and (3.2) to get (with the notation $\tau(Y)$ for $\tau(Y(x, t))$ )

$$
E=\int_{\Omega} W\left(\operatorname{tr} B, \operatorname{tr} B^{-1}, \tau(Y)^{T} \nabla Y^{-T}(x, t) \nabla Y^{-1}(x, t) \tau(Y), \tau(Y)^{T} \nabla Y(x, t) \nabla Y^{T}(x, t) \tau(Y)\right) \mathrm{d} x
$$

or, equivalently,

$$
E=\int_{\Omega} W\left(\operatorname{tr} B, \operatorname{tr} B^{-1},\left|\nabla Y^{-1}(x, t) \tau(Y)\right|^{2},\left|\nabla Y^{T}(x, t) \tau(Y)\right|^{2} \mathrm{~d} x\right.
$$

As a result, an incompressible elastic material with transverse isotropy symmetry can be represented by a stored energy of the form

$$
E=\frac{1}{2} \int_{\Omega} W\left(\left|\nabla Y^{-1}\right|^{2},|\nabla Y|^{2},\left|\nabla Y^{T} \tau(Y)\right|^{2},\left|\nabla Y^{-1} \tau(Y)\right|^{2}\right) \mathrm{d} x
$$

where $W$ is a given function depending on 4 parameters. To take the time derivative of this expression, we first observe that $\tau(Y)$ satisfies a transport equation, along the velocity field $u$, from which we readily get

$$
\begin{array}{r}
\left(\left|\nabla Y^{T} \tau\right|^{2}\right)_{t}+u \cdot \nabla\left(\left|\nabla Y^{T} \tau\right|^{2}\right)+2\left(\nabla Y^{T} \tau \otimes \nabla Y^{T} \tau\right): \nabla u=0 \\
\left(\left|\nabla Y^{-1} \tau\right|^{2}\right)_{t}+u \cdot \nabla\left(\left|\nabla Y^{-1} \tau\right|^{2}\right)-2\left(\nabla Y^{-1} \tau \otimes \nabla Y^{-1} \tau\right): \nabla u=0 .
\end{array}
$$

Combined with equation (3.6) for $\left|\nabla Y^{-1}\right|^{2}$ and $|\nabla Y|^{2}$, this leads to the following expression for the stress

$$
\sigma=-p \mathbb{I}+\frac{\partial W}{\partial a} B-\frac{\partial W}{\partial b} B^{-1}-\frac{\partial W}{\partial c} \nabla Y^{T} \tau \otimes \nabla Y^{T} \tau+\frac{\partial W}{\partial d} \nabla Y^{-1} \tau \otimes \nabla Y^{-1} \tau .
$$

From now on we choose this preferred direction as being $\tau(y)=e_{3}$, the third basis vector. This simple case will permit to identify the mechanical meaning of different terms. The case of material with non-constant preferred direction will be seen next.

Let us write the different terms appearing in this expression in a more level-set flavored way. Since we chose $\tau=e_{3}$, from (3.8) we have

$$
\nabla Y^{T} \tau=\nabla Y_{3} \text { and } \nabla Y^{-1} \tau=\nabla X e_{3}=X_{\xi_{3}}=\nabla Y_{1} \times \nabla Y_{2}
$$

Combined with the expressions of $B$ and $B^{-1}$ previously obtained, this gives the following general form for the stress of this anisotropic medium:

$$
\begin{aligned}
\sigma=-p \mathbb{I} & -\alpha_{12} \sum_{i=1}^{2} \nabla Y_{i} \otimes \nabla Y_{i}-\alpha_{3} \nabla Y_{3} \otimes \nabla Y_{3} \\
& +\beta_{12}\left[\left(\nabla Y_{2} \times \nabla Y_{3}\right) \otimes\left(\nabla Y_{2} \times \nabla Y_{3}\right)+\left(\nabla Y_{3} \times \nabla Y_{1}\right) \otimes\left(\nabla Y_{3} \times \nabla Y_{1}\right)\right] \\
& +\beta_{3}\left(\nabla Y_{1} \times \nabla Y_{2}\right) \otimes\left(\nabla Y_{1} \times \nabla Y_{2}\right)
\end{aligned}
$$

where $\alpha_{12}, \alpha_{3}, \beta_{12}, \beta_{3}$ are coefficients depending on $|\nabla Y|^{2},\left|\nabla Y^{-1}\right|^{2},\left|\nabla Y_{3}\right|^{2},\left|\nabla Y_{1} \times \nabla Y_{2}\right|^{2}$. We have thus established the following result: 
Proposition 3.2. The deformation of a transverse isotropic elastic material, with preferred direction $e_{3}$ at time $t=0$, is governed by the following equations:

$$
\begin{cases}\rho\left(u_{t}+u \cdot \nabla u\right)-\operatorname{div} \sigma=f, & \text { on } \Omega \times] 0, T[, \\ \operatorname{div} u=0, & \text { on } \Omega \times] 0, T[, \\ Y_{t}+u \cdot \nabla Y=0, & \text { on } \Omega \times] 0, T[, \\ u=0, & \text { on } \partial \Omega \times] 0, T[, \\ u=u_{0}, \quad Y=i d, & \text { on } \Omega \times\{0\},\end{cases}
$$

where $\sigma$ is given by (3.10) and the $\alpha_{i}$ are functions of $|\nabla Y|^{2},\left|\nabla Y^{-1}\right|^{2},\left|\nabla Y_{3}\right|^{2},\left|\nabla Y_{1} \times \nabla Y_{2}\right|^{2}$ defined as partial derivatives of an energy function $W$.

We conclude the analysis in this simple case with a few remarks concerning the links between our formulation and the works of Ogden. In [13], the two anisotropic invariants are denoted by $I_{4}:=\tau^{T} C \tau$ and $I_{5}:=\tau^{T} C^{2} \tau$. $I_{4}$ corresponds exactly to $\left|\nabla Y_{1} \times \nabla Y_{2}\right|^{2}$, while $I_{5}$ may be expressed in terms of $\left|\nabla Y_{3}\right|^{2}$ and $\left|\nabla Y_{1} \times \nabla Y_{2}\right|^{2}$. After some calculations, it can be proved that the invariant $I_{4}$ measures the stretching along the curve $\left\{Y_{1}=0\right\} \cap\left\{Y_{2}=0\right\}$, an interpretation in agreement with [11]. The fact that $\left|\nabla Y_{3}\right|^{2}$ measures the stretching along $\nabla Y_{3}$ is obtained easily by differentiating the transport equation verified by $Y_{3}$ and in the incompressible case, it was shown in [5] that this corresponds also to the area change of $\left\{Y_{3}=0\right\}$. In [13] it is stated that no simple interpretation of $I_{5}$ is known in general, since it mixes this stretching in the normal direction and along the material vertical lines. In our Eulerian formulation, it appears that a more convenient choice for $I_{5}$, and which gives an equivalent set of invariants, would be $\tau^{T} C^{-1} \tau$. This invariant has a clear mechanical meaning. Indeed it measures the stretching in the direction normal to $Y_{3}=0$, a direction which is in general distinct from $\nabla Y_{1} \times \nabla Y_{2}$, in the case when transverse shear occurs.

\subsubsection{Transversely isotropic elasticity: level set formulation in the general case}

Let us finally derive the form taken by the stress when the preferred direction is not constant. In this Eulerian approach of elasticity, the level-set method is the natural way to describe this direction. Let $\varphi_{0}$ be a distance function to an hypersurface $\left(\left|\nabla \varphi_{0}\right|=1\right)$ such that the preferred direction is given by $\tau(y)=\nabla \varphi_{0}(y)$. Let $\varphi(x, t)=\varphi_{0}(Y(x, t))$ be the corresponding transported function; one has

$\nabla Y^{T} \tau(Y)=\nabla Y^{T} \nabla \varphi_{0}(Y)=\nabla \varphi(x, t), \nabla Y^{-1} \tau(Y)=\nabla Y^{-1} \nabla \varphi_{0}(Y)=\nabla Y^{-1} \nabla Y^{-T} \nabla Y^{T} \nabla \varphi_{0}(Y)=B \nabla \varphi(x, t)$

Note that $\nabla \varphi$, unlike $B \nabla \varphi$, does not remain the material preferred direction, but rather is orthogonal to the unpreferred two other directions. Thus the two measures of deformations are $\left|\nabla Y^{T} \tau\right|=|\nabla \varphi|$ and $\left|\nabla Y^{-1} \tau\right|=$ $|B \nabla \varphi|$. The former is the stretching along the normal $\{\lambda \nabla \varphi, \lambda \in \mathbb{R}\}$ to the unpreferred directions. In the incompressible case this corresponds also to the change of area of the surface $\{\varphi=0\}$. The latter is the stretching in the preferred direction. Note that this subtle distinction is made possible by the use of a level-set function, which also simplifies dramatically the (equivalent) form of the resulting stress (compared to Eq. (73) of [13], p. 79, or Eq. (6.209) in [7], p. 269), which now takes the following simple form:

$$
\sigma=-p \mathbb{I}+\frac{\partial W}{\partial a} B-\frac{\partial W}{\partial b} B^{-1}-\frac{\partial W}{\partial c} \nabla \varphi \otimes \nabla \varphi+\frac{\partial W}{\partial d} B \nabla \varphi \otimes B \nabla \varphi
$$


We have established the following result:

Proposition 3.3. The deformation of a transverse isotropic elastic material, with initial preferred direction $\nabla \varphi_{0}(y)$, where $\varphi_{0}$ is a distance function, is governed by the following equations:

$$
\begin{cases}\rho\left(u_{t}+u \cdot \nabla u\right)-\operatorname{div} \sigma=f, & \text { on } \Omega \times] 0, T[, \\ \operatorname{div} u=0, & \text { on } \Omega \times] 0, T[, \\ Y_{t}+u \cdot \nabla Y=0, & \text { on } \Omega \times] 0, T[, \\ \varphi(x, t)=\varphi_{0}(Y(x, t)), & \text { on } \Omega \times] 0, T[, \\ u=0, & \text { on } \partial \Omega \times] 0, T[, \\ u=u_{0}, \quad Y=i d, & \text { on } \Omega \times\{0\},\end{cases}
$$

where $\sigma$ is given by (3.11) and the $\alpha_{i}$ are functions of $|\nabla Y|^{2},\left|\nabla Y^{-1}\right|^{2},|\nabla \varphi|^{2},|B \nabla \varphi|^{2}$ defined as partial derivatives of an energy function $W$.

Remark 3.4. (i) We could recover the form of Ogden and Holzapfel by taking their $I_{5}$ invariant based on $C^{2}$ instead of using $C^{-1}$. This would correspond in the above formulation to changing $\left|\nabla Y^{-1} \tau(Y)\right|$ into $\left|\nabla Y^{-T} \nabla Y^{-1} \tau(Y)\right|$ and make the level set formulation slightly less direct.

(ii) If we consider an initial level-set function which is not a distance, and define $\tau(y)=\frac{\nabla \varphi_{0}}{\left|\nabla \varphi_{0}\right|}(y)$, then the corresponding part of the above stress has to be divided by $\left|\nabla \varphi_{0}(Y(x, t))\right|$.

(iii) We could have derived directly the stress from the fact that $\nabla \varphi$ and $B \nabla \varphi$ satisfy similar stretching equations, as seen from (3.3), namely

$$
\nabla \varphi_{t}+u \cdot \nabla(\nabla \varphi)=-\nabla u^{T} \nabla \varphi \quad(B \nabla \varphi)_{t}+u \cdot \cdot \nabla(B \nabla \varphi)=\nabla u B \nabla \varphi .
$$

\subsubsection{Some comments on the $2 D$ case}

The dimension 2 case turns out to be simpler. Indeed for a unit vector $\tau$,

$$
\begin{gathered}
\nabla Y=\left(\begin{array}{ll}
Y_{1, x_{1}} & Y_{1, x_{2}} \\
Y_{2, x_{1}} & Y_{2, x_{2}}
\end{array}\right) \quad \nabla Y^{-1}=\left(\begin{array}{cc}
Y_{2, x_{2}} & -Y_{1, x_{2}} \\
-Y_{2, x_{1}} & Y_{1, x_{1}}
\end{array}\right) \\
\nabla Y^{T} \tau=\left(\begin{array}{c}
\tau_{1} Y_{1, x_{1}}+\tau_{2} Y_{2, x_{1}} \\
\tau_{1} Y_{1, x_{2}}+\tau_{2} Y_{2, x_{2}}
\end{array}\right) \quad \nabla Y^{-1} \tau=\left(\begin{array}{c}
\tau_{1} Y_{2, x_{2}}-\tau_{2} Y_{1, x_{2}} \\
-\tau_{1} Y_{2, x_{1}}+\tau_{2} Y_{1, x_{1}}
\end{array}\right) .
\end{gathered}
$$

Thus $|\nabla Y|^{2}=\left|\nabla Y^{-1}\right|^{2}$ and $\left|\nabla Y^{-1} \tau\right|^{2}+\left|\nabla Y^{T} \tau\right|^{2}=|\nabla Y|^{2}$. A generic energy function for a transverse isotropic $2 D$ incompressible elastic material is therefore given by

$$
E=\int_{\Omega} \hat{W}\left(|\nabla \varphi|^{2},|B \nabla \varphi|^{2}\right) \mathrm{d} x
$$

leading to the following stress:

$$
\sigma=-p \mathbb{I}-\frac{\partial \hat{W}}{\partial a} \nabla \varphi \otimes \nabla \varphi+\frac{\partial \hat{W}}{\partial b} B \nabla \varphi \otimes B \nabla \varphi .
$$

In general it is not possible to find a globally defined distance function $\psi_{0}$ such that $\nabla \psi_{0} \cdot \nabla \varphi_{0}=0$, especially when the zero level set of $\varphi_{0}$ represents a closed curve. But it is possible at least locally to find such a function $\psi_{0}$ (see [5]). Then, if $\psi$ is the transported function from $\psi_{0}$, one has

$$
\nabla Y^{-1} \nabla \varphi_{0}(Y)=\left(\nabla Y^{T} \nabla \psi_{0}(Y)\right)^{\perp}=\nabla \psi(x)^{\perp}
$$


and thus

$$
\nabla Y^{-1} \nabla \varphi_{0}(Y) \otimes \nabla Y^{-1} \nabla \varphi_{0}(Y)=|\nabla \psi|^{2} \mathbb{I}-\nabla \psi \otimes \nabla \psi
$$

The diagonal part being absorbed by the pressure, the following simple form of stress is therefore obtained

$$
\sigma=-q \mathbb{I}-\frac{\partial \hat{W}}{\partial a} \nabla \varphi \otimes \nabla \varphi-\frac{\partial \hat{W}}{\partial b} \nabla \psi \otimes \nabla \psi
$$

Again, we stress that in general $\psi$ is only locally defined, so that the form (3.13) should be used instead of this one. The above expression is provided as it gives a more appealing insight into what the second part of stress in (3.13) does represent, that is, a stretching along the curve $\{\psi=0\}$. Note that this model, except when the coefficients are constants, has a non trivial response to a simple shear (i.e. it is not reduced to a gradient term). One could be convinced by simply considering $\tau=e_{2}$, the velocity field $u\left(x_{1}, x_{2}\right)=\left(-x_{2}, 0\right)$ which corresponds to $Y_{1}=x_{1}-x_{2}$ t and $Y_{2}=x_{2}$.

In the next section we will compare the stress derived in (3.13) with the one obtained for an immersed membrane. However the latter was derived from (2.4) which is written as a function of $|\nabla \varphi|$ rather than its square. In the next subsection we consider a form of the stress obtained from an energy

$$
E=\int_{\Omega} W(|\nabla \varphi|,|B \nabla \varphi|) \mathrm{d} x
$$

and which is given by

$$
\sigma=-p \mathbb{I}-\alpha_{1}(|\nabla \varphi|,|B \nabla \varphi|) \frac{\nabla \varphi \otimes \nabla \varphi}{|\nabla \varphi|}+\alpha_{2}(|\nabla \varphi|,|B \nabla \varphi|) \frac{B \nabla \varphi \otimes B \nabla \varphi}{|B \nabla \varphi|} .
$$

\subsection{Incompressible elastic body immersed in a fluid}

We are now in a position to derive generic fluid-structure interaction Eulerian models relying on level set functions. It essentially suffices to introduce an additional level set function to capture the fluid-solid interface and switch between the fluid and solid stress forms.

Let us denote by $\Omega_{F}(t)$ and $\Omega_{S}(t)$ respectively the moving fluid and solid domains and by $\Sigma(t)$ their interface. Assume that the interface $\Sigma(0)$ coincides with the zero level set of a smooth function $\Psi_{0}$ and that $\Psi_{0}$ is positive inside $\Omega_{S}(0)$. The densities of the fluid and solid are denoted by $\rho_{F}$ and $\rho_{S}$. In the sequel, $H$ denotes the Heaviside function. The following result gives a general level set formulation for fluid-structure interaction:

Proposition 3.5. With the above notations, a generic fluid-structure interaction model is given by the following system of equations:

$$
\begin{cases}\rho(\Psi)\left(u_{t}+u \cdot \nabla u\right)-\operatorname{div}\left(H(\Psi) \sigma_{S}+(1-H(\Psi)) \sigma_{F}\right)=f & \text { on } \Omega \times] 0, T[, \\ \operatorname{div} u=0 & \text { in } \Omega \times] 0, T[, \\ Y_{t}+u \cdot \nabla Y=0 & \text { in } \Omega \times] 0, T[, \\ \varphi(x, t)=\varphi_{0}(Y(x, t)), \Psi(x, t)=\Psi_{0}(Y(x, t)) & \text { in } \Omega \times] 0, T[, \\ u=0 & \text { in } \partial \Omega \times] 0, T[, \\ u=u_{0}, \quad \Psi=\Psi_{0}, \quad Y=I d & \text { in } \Omega \times\{0\} .\end{cases}
$$

In the above system, $\rho(\Psi)=H(\Psi) \rho_{S}+(1-H(\Psi)) \rho_{F}, \sigma_{F}=-p I+2 \mu D(u)$ is the fluid stress tensor, and $\sigma_{S}$ is the elastic stress tensor. In the case of isotropic elasticity in $\Omega_{S}, \sigma_{S}$ is given by (3.7). For a transverse isotropic solid with preferred direction at time zero $\tau(y)=\nabla \varphi_{0}(y), \sigma_{S}$ is given by $(3.15)$ with $\varphi(x, t)=\varphi_{0}(Y(x, t))$.

This result merely states that the momentum conservation equation implicitly translates the fact that the deformations satisfy the flow equations in the fluid, the elasticity equations in the solid and fulfill continuity of velocity and stresses at the fluid-solid interface. 


\subsection{Asymptotic toward the immersed membrane level-set model in dimension 2}

We consider in this section a thin elastic body of width $2 \varepsilon$, in the sense that it initially lies between the level sets $\varphi_{0}=-\varepsilon$ and $\varphi_{0}=\varepsilon$ for a given smooth function $\varphi_{0}$. We also assume that it is transversely isotropic, with preferred direction $\nabla \varphi_{0}$. We set

$$
\zeta(r)=H(r+1)-H(r-1)
$$

( $\zeta$ is the top-hat cut-off). With these notations, the momentum conservation equation in Proposition 3.5 takes the form

where

$$
\rho_{\varepsilon}(\varphi)\left(u_{t}+u \cdot \nabla u\right)-\operatorname{div} \sigma_{\varepsilon}=f
$$

$$
\sigma_{\varepsilon}=-p \mathbb{I}+2\left(1-\zeta\left(\frac{\varphi}{\varepsilon}\right)\right) \mu D(u)+\zeta\left(\frac{\varphi}{\varepsilon}\right)\left(\beta_{1}^{\varepsilon} B-\beta_{2}^{\varepsilon} B^{-1}-\alpha_{1}^{\varepsilon} \frac{\nabla \varphi \otimes \nabla \varphi}{|\nabla \varphi|}+\alpha_{2}^{\varepsilon} \frac{B \nabla \varphi \otimes B \nabla \varphi}{|B \nabla \varphi|}\right) .
$$

Note that for this model, one can derive the following energy equality:

$$
\begin{array}{r}
\frac{1}{2} \int_{\Omega} \rho_{\varepsilon}(\varphi) u^{2}(x, t) \mathrm{d} x+\int_{\Omega} W\left(|\nabla Y|^{2},\left|\nabla Y^{-1}\right|^{2},|\nabla \varphi|,|B \nabla \varphi|\right) \zeta\left(\frac{\varphi}{\varepsilon}\right) \mathrm{d} x+\int_{0}^{t} \int_{\Omega} \mu\left(1-\zeta\left(\frac{\varphi}{\varepsilon}\right)\right)|\nabla u|^{2} \mathrm{~d} x= \\
\int_{\Omega} f u \mathrm{~d} x+\frac{1}{2} \int_{\Omega} \rho_{\varepsilon}\left(\varphi_{0}\right) u_{0}^{2}(x) \mathrm{d} x+\int_{\Omega} W(1,1,1,1) \zeta\left(\frac{\varphi_{0}}{\varepsilon}\right) \mathrm{d} x
\end{array}
$$

In general $W(1,1,1,1) \neq 0$ except if the initial state is at rest.

Remark 3.6. We observe that this energy is very close to the membrane model, up to extra level-set functions and the lack of diffusion in the solid domain. However, if we consider a viscoelastic solid, that is if we add in (3.17) to the coefficient $2\left(1-\zeta\left(\frac{\varphi}{\varepsilon}\right)\right) \mu$ a small diffusion coefficient in the solid, e.g. $\varepsilon\left(1-\zeta\left(\frac{\varphi}{\varepsilon}\right)\right)$, then a generalization of the existence Theorem 2.2 stated in the preceding section is straightforward.

Our purpose is now to study formally the asymptotic behavior of the above fluid-structure problem when the thickness of the structure goes to zero, compared to the immersed interface model. For simplicity we will consider the $2 D$ case.

To begin with, the correct ansatz for the density to recover a membrane with surface mass as given in the model (2.9) is to assume that

Next, the stress tensor (3.17) is given by

$$
\rho_{\varepsilon}(\varphi)=\frac{1}{\varepsilon} \zeta\left(\frac{\varphi}{\varepsilon}\right) \lambda_{\theta}+\left(1-\zeta\left(\frac{\varphi}{\varepsilon}\right)\right) \rho_{F}
$$

$$
\sigma_{\varepsilon}=-p \mathbb{I}+2\left(1-\zeta\left(\frac{\varphi}{\varepsilon}\right)\right) \mu D(u)-\zeta\left(\frac{\varphi}{\varepsilon}\right)\left(\alpha_{1}^{\varepsilon} \frac{\nabla \varphi \otimes \nabla \varphi}{|\nabla \varphi|}-\alpha_{2}^{\varepsilon} \frac{B \nabla \varphi \otimes B \nabla \varphi}{|B \nabla \varphi|}\right),
$$

and must be compared to the stress tensor corresponding to an immersed elastic interface. In the case where curvature forces were neglected, recall that this stress tensor was given by

$$
\sigma_{m}^{\varepsilon}=-q \mathbb{I}+2 \mu D(u)-E^{\prime}(|\nabla \varphi|) \frac{\nabla \varphi \otimes \nabla \varphi}{|\nabla \varphi|} \frac{1}{\varepsilon} \zeta\left(\frac{\varphi}{\varepsilon}\right) .
$$

The first fact is that the non-viscous zone disappears as $\varepsilon \rightarrow 0$ since $1-\zeta\left(\frac{\varphi}{\varepsilon}\right) \rightarrow 0$. Thus we recover the same fluid stress tensor. To understand the limiting process concerning the other terms, we recall that $\alpha_{1}$ (resp. $\left.\alpha_{2}\right)$ is the derivative of the energy density with respect to a stretching in the direction $\{\varphi=0\}$ (resp. in a direction transverse to $\{\varphi=0\}$ ), which is recorded by $|\nabla \varphi|$ (resp. $|B \nabla \varphi|)$. In our case $\alpha_{1}$ is a measure of how fast the density of energy increases when the body is elongated along $\{\varphi=0\}$. This term must increase when the width of the body decreases, since a thiner strip of elastic body is more deformed than a thicker one under the same stress. 
Thus we take $\alpha_{1}$ proportional to $\frac{1}{2 \alpha(\varepsilon)}$. On the contrary, as the material is incompressible, a loss of width in one direction corresponds to a gain in the other, thus by the same argument $\alpha_{2}$ should be proportional to the width. This discussion leads to the following ansatz:

$$
\alpha_{1}=\frac{1}{2 \varepsilon} \alpha_{1}^{0}+\alpha_{1}^{1}+2 \alpha_{1}^{2} \varepsilon+O\left(\alpha(\varepsilon)^{2}\right) \quad \alpha_{2}=2 \alpha_{2}^{0} \varepsilon+O\left(\varepsilon^{2}\right) .
$$

Inserting this into the above stress tensor shows that formally (that is under the assumption that $p$ and $u$ are bounded in strong enough norms) to the first order in $\varepsilon$,

$$
\sigma_{\varepsilon}=-p \mathbb{I}+2 \mu D(u)-\alpha_{1}^{0} \frac{\nabla \varphi \otimes \nabla \varphi}{|\nabla \varphi|} \frac{1}{\varepsilon} \zeta\left(\frac{\varphi}{\varepsilon}\right)
$$

which is the immersed boundary model, provided $\alpha_{1}^{0}(|\nabla \varphi|,|B \nabla \varphi|)=E^{\prime}(|\nabla \varphi|)$. The other terms are expected to account for higher order curvature effects and will not be considered in this present work. Presumably one should be able to express membrane and flexural stress for an elastic curve (resp. surface) using two preferred directions as above instead of a single one, and by doing this gaining an order of derivative in the final expression. This is a direction we wish to pursue.

\section{Numerical iLlustrations}

In this section we compare the generic fluid-structure coupling model we just developed with our reference membrane model. We consider the same test case we used in $[5,8]$ to validate the membrane model against immersed boundary methods. In this test, a circle of radius 0.5 is stretched into an elastic ellipse with major axis 1.5 and minor axis 1 (this corresponds to a stretching of about 1.2625). This mass-less ellipse is immersed in a fluid. We study its relaxation to a circle up to $t=20$, by tracing the horizontal and vertical radii with respect to time. Note that, by volume conservation, the limit circle must have a radius equal to $\sqrt{(0.75 \times 0.5)} \simeq 0.6124$. This value is greater than 0.5 , which means that the equilibrium is a stretched configuration with a pressure gradient across the interface. For the membrane case, accurate pressure plots for $t=0.2$ and $t=2.2$ units of time are available. The computational box is a square of size $2 \times 2$.

We first need to comment on how the initial stretching of the membrane is introduced in the model. There are two ways to proceed: in the first one we would introduce a function $\alpha_{1}(|\nabla \varphi|)=\lambda(|\nabla \varphi|-1)$ and initialize $\varphi$ so that it carries the initial stretching, as described in [5]. Thus the elastic tensor is

$$
\lambda(|\nabla \varphi|-1) \frac{\nabla \varphi \otimes \nabla \varphi}{|\nabla \varphi|} .
$$

This corresponds exactly to the membrane formulation, except that it is written in divergence form whereas in the calculations of [5] the divergence was developed to yield a force with normal and tangential components.

In the second way to prescribe initial stretching, we substract from the current tensor of deformation the initial one. More precisely, our elastic tensor is now

$$
\lambda\left(\frac{\nabla \varphi \otimes \nabla \varphi}{|\nabla \varphi|}-\frac{\nabla \varphi_{0} \otimes \nabla \varphi_{0}}{E_{0}}\right),
$$

where $E_{0}$ is the initial stretching. This form turns out to be numerically more stable than the other when written in divergence form and therefore will be chosen in the sequel. Note however that the first form gives similar results if the divergence is expanded, since it becomes exactly the membrane model. As the second way to impose the initial stretching was more direct and stable, we used that one in all the numerical tests presented here.

In a first simulation we ran the isotropic fluid-structure model, i.e. using the stress (3.17) with $\alpha_{1}=\alpha_{2}=0$. In dimension $2, B$ and $B^{-1}$ are linked by the Cayley-Hamilton theorem thus one needs only to consider one 


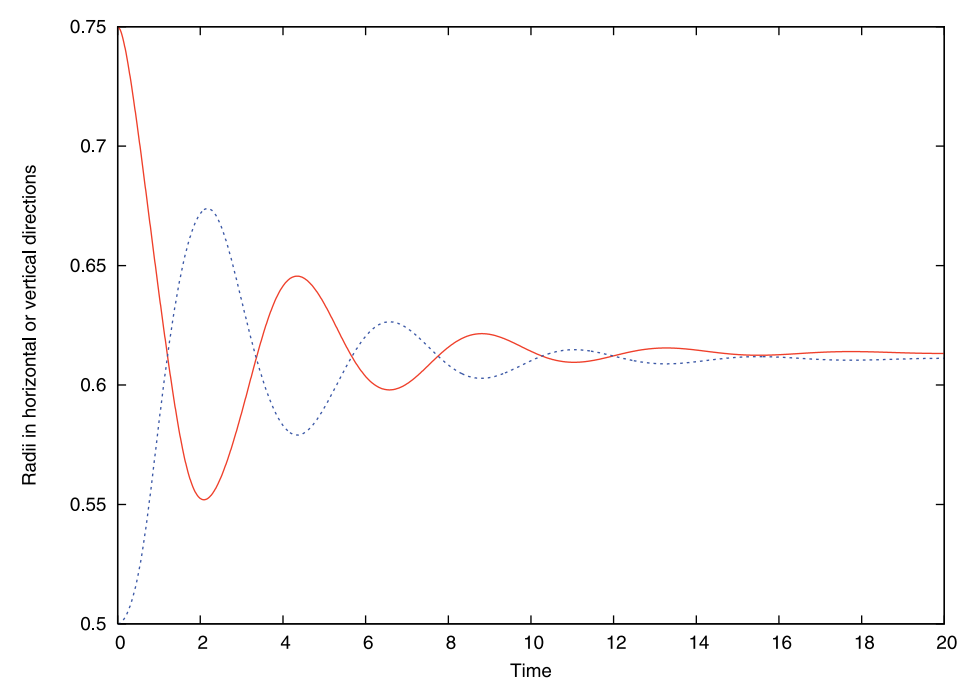

FiguRE 1. Reference membrane relaxation. Time evolution of long and small axis.

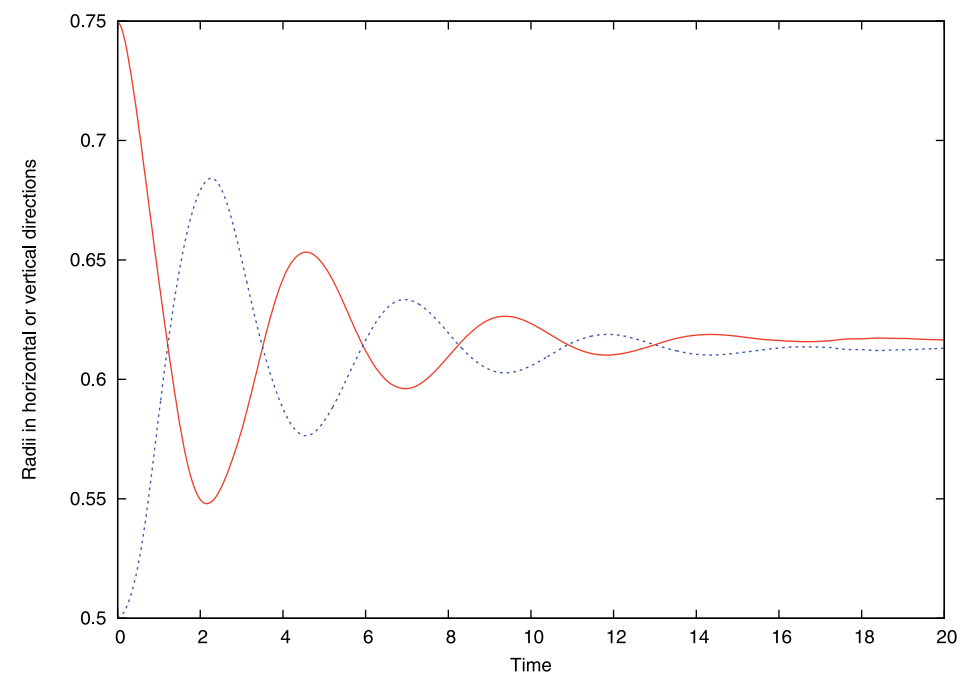

Figure 2. Relaxation of an isotropic thin elastic ellipse. Time evolution of long and small axis.

of the two coefficients $\beta_{i}$. We chose $\beta_{1}$, but results are identical with $\beta_{2}$ up to the pressure. Computations were made on a $256 \times 256$ grid, and $\varepsilon=2 \mathrm{~d} x$. The coefficient $\beta_{1}$ was tuned so that the first time at which the ellipse is a circle (i.e. the vertical and horizontal radii are equal) is the same as for the membrane reference case. The dynamics in the membrane (Fig. 1) and isotropic solid cases (Fig. 2) exhibit similar qualitative behaviour.

Next we consider the anisotropic case, i.e. the stress given in (3.18) with $\alpha_{2}=0$. We observed that, in absence of dissipation in the elastic domain, this configuration is unstable. Indeed in the isotropic case, both directions of the elastic solid were subject to elasticity while in this anisotropic setting, somehow we canceled the reaction to deformations in one of these. If we add this dissipation the dynamics as well as the pressure profiles are similar to those of the membrane case.

To provide with numerical evidence of the convergence of the anisotropic model towards the membrane model, we consider three thickness in the former, with 5, 3 and 2 grid points in the elastic part (plus the 

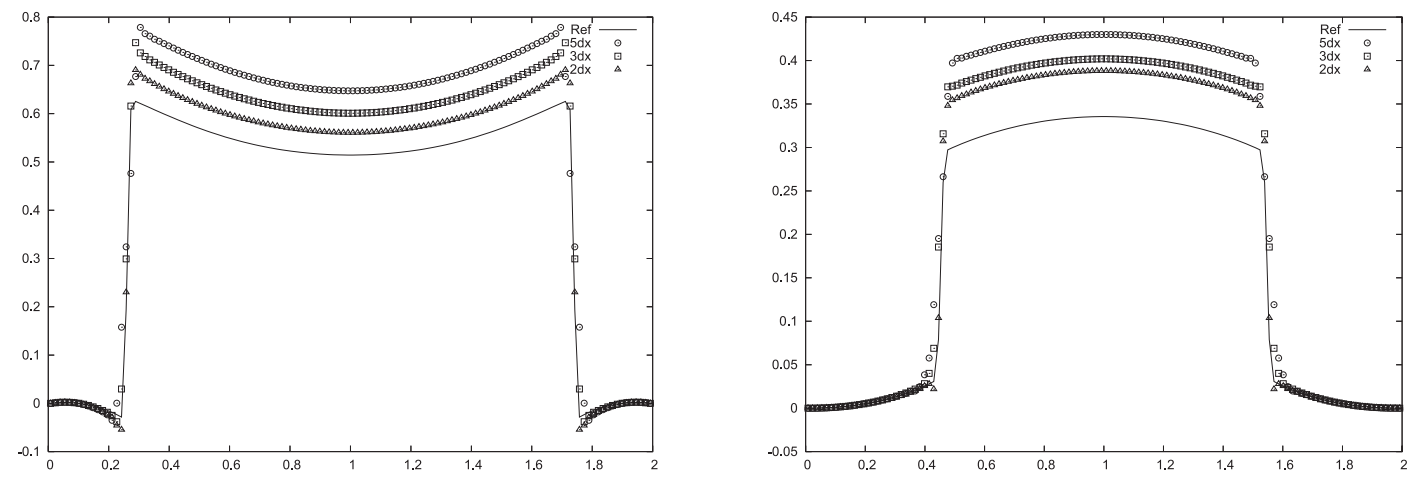

Figure 3. Convergence of pressure profiles at $t=0.2$ (left) and $t=2.2$ (right) towards the reference profile (membrane) for different values of $\varepsilon$.

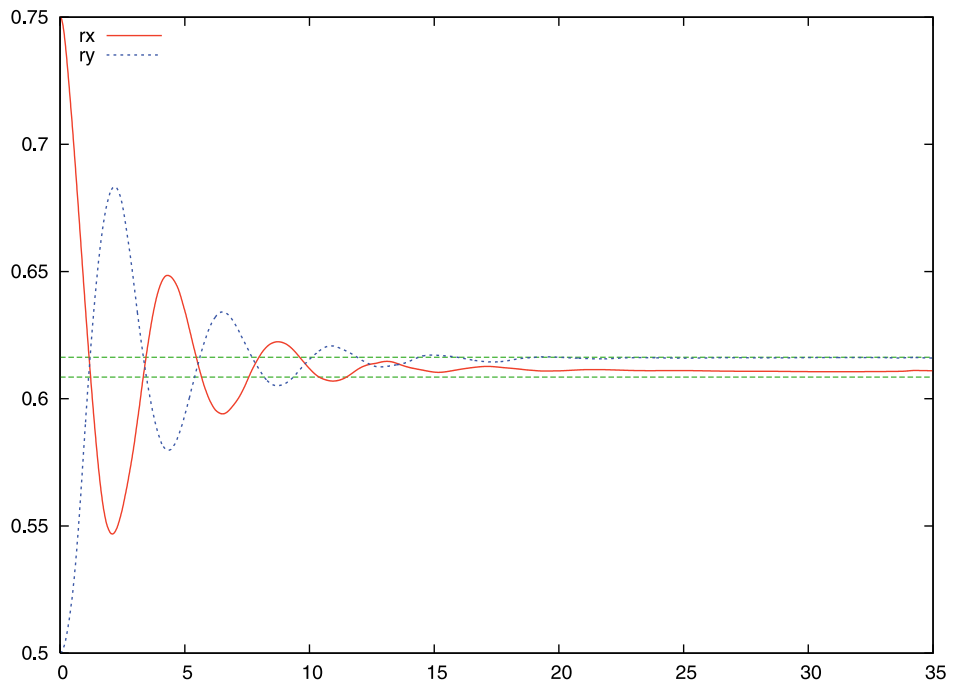

Figure 4. Relaxation of an anisotropic thin elastic ellipse. Time evolution of long and small axis. The horizontal dashed lines represent the values $r=0.6124 \pm \Delta x / 2$.

spreading zone), and keep adjusting the stiffness so that the first circle configuration occurs at the same time in all tests. We compare in Figure 3 the pressure plots at $t=0.2$ and $t=2.2$ with the reference plot given by the membrane model. Note that we are interested in the convergence of the continuous models. After numerical discretization this convergence can clearly only be observed up to the discretization level. We thus considered for this convergence study a rather fine grid.

On Figure 4 we reproduce the test of a relaxing ellipse in this anisotropic case. The number of grid points in each direction is 256 and $\varepsilon=2 \Delta x$. We observe a very good agreement with the membrane and isotropic cases in Figures 1 and 2. To illustrate the excellent volume conservation which is achieved by the projection method used on a MAC mesh, we ran the simulation further in time. The volume conservation is illustrated by the fact that the long and small axes converge to steady values, which are equal, up to one discretization point, to the radius of the circle with the same volume as the initial ellipse. The typical volume loss at $t=35$, which corresponds to about 140000 iteration steps for $n=256$, is $0.5 \%$. We refer to [5] for a precise volume conservation study with respect to the discretization step in the case of an elastic membrane. 


\section{Conclusion}

We have derived from energy principles a class of Eulerian models based on level set functions for fluidstructure interaction. The elastic stresses are recovered from level set functions by writing the Cauchy-Green tensor in terms of the backward trajectories. This framework covers isotropic as well are anisotropic elasticity. It enables to deal with complex fluid-structure problems, which would be time consuming by more traditional ALE approach, using off-the shelf finite difference solvers. In the case of an elastic membrane immersed in a viscous fluid we are able to prove local existence of strong solutions. Future plans include combining these methods with Adaptive Mesh Refinement techniques for finite-difference solvers.

Acknowledgements. This work was completed while the second author was visiting the School of Mathematics of the University of Minnesota in Minneapolis. It was supported by the French Ministry of Education through ACI grant 04-5 290 and by ANR through grant 06-BLAN-0306.

\section{REFERENCES}

[1] M. Boulakia, Modélisation et analyse mathématique de problèmes d'interaction fluide-structure. Ph.D. thesis, Université de Versailles, France (2004).

[2] L. Caffarelli, R. Kohn and L. Nirenberg, Partial regularity of suitable weak solutions of the Navier-Stokes equations. Comm. Pure Appl. Math. 35 (1982) 771.

[3] P.G. Ciarlet, Elasticité tridimensionnelle. Masson (1985).

[4] G.-H. Cottet and E. Maitre, A level-set formulation of immersed boundary methods for fluid-structure interaction problems. C. R. Acad. Sci. Paris, Ser. I 338 (2004) 581-586.

[5] G.-H. Cottet and E. Maitre, A level-set method for fluid-structure interactions with immersed surfaces. Math. Models Methods Appl. Sci. 16 (2006) 415-438.

[6] G.-H. Cottet, E. Maitre and T. Milcent, An Eulerian method for fluid-structure interaction with biophysical applications, in European Conference on Computational Fluid Dynamics, ECCOMAS CFD 2006, P. Wesseling, E. Oñate and J. Périaux Eds., TU Delft, The Netherlands (2006).

[7] G.A. Holzapfel, Nonlinear solid mechanics: a continuum approach for engineering. Wiley (2000).

[8] L. Lee and R.J. Leveque, An immersed interface method for incompressible Navier-Stokes equations. SIAM J. Sci. Comp. 25 (2003) 832-856.

[9] E. Maitre, T. Milcent, G.-H. Cottet, A. Raoult and Y. Usson, Applications of level set methods in computational biophysics. Math. Comput. Model. (to appear).

[10] E. Maitre, C. Misbah and A. Raoult, Comparison between advected-field and level-set methods in the study of vesicle dynamics. (In preparation).

[11] J. Merodio and R.W. Ogden, Mechanical response of fiber-reinforced incompressible non-linearly elastic solids. Int. J. Nonlinear Mech. 40 (2005) 213-227.

[12] R.W. Ogden, Non-linear elastic deformations. Dover Publications (1984).

[13] R.W. Ogden, Nonlinear elasticity, anisoptropy, material staility and residual stresses in soft tissue, in Biomechanics of Soft Tissue in Cardiovascular Systems, G.A. Holzapfel and R.W. Ogden Eds., CISM Course and Lectures Series 441, Springer, Wien (2003) 65-108.

[14] S. Osher and R.P. Fedkiw, Level set methods and Dynamic Implicit Surfaces. Springer (2003).

[15] C.S. Peskin, The immersed boundary method. Acta Numer. 11 (2002) 479-517.

[16] P. Smereka, The numerical approximation of a delta function with application to level-set methods. J. Comp. Phys. 211 (2003) $77-90$.

[17] V.A. Solonikov, Estimates for solutions of nonstationary Navier-Stokes equations. J. Soviet Math. 8 (1977) 467-529.

[18] M. Sy, D. Bresch, F. Guillén-González, J. Lemoine and M.A. Rodríguez-Bellido, Local strong solution for the incompressible Korteweg model. C. R. Acad. Sci. Paris, Ser. I 342 (2006) 169-174.

[19] H. Triebel, Interpolation theory, function spaces, differential operators. North-Holland (1978). 